\title{
SYMMETRIES AND ADJUNCTION INEQUALITIES FOR KNOT FLOER HOMOLOGY
}

\author{
BIJAN SAHAMIE
}

\begin{abstract}
We derive symmetries and adjunction inequalities of the knot Floer homology groups which appear to be especially interesting for homologically essential knots. Furthermore, we obtain an adjunction inequality for cobordism maps in knot Floer homologies. We demonstrate the adjunction inequalities and symmetries in explicit calculations which recover some of the main results from [1] on longitude Floer homology and also give rise to vanishing results on knot Floer homologies. Furthermore, using symmetries we prove that the knot Floer homology of a fiber distinguishes $\mathbb{S}^{2} \times \mathbb{S}^{1}$ from other $\mathbb{S}^{1}$-bundles over surfaces.
\end{abstract}

\section{IntRoduction}

Heegaard Floer homology was introduced by Peter Ozsváth and Zoltan Szabó in [12] (see [18] for a detailed introduction) and has turned out to be a useful tool in the study of low-dimensional topology. They also defined variants of this homology theory which are topological invariants of a pair $(Y, K)$ where $Y$ is a closed, oriented 3-manifold and $K \subset Y$ a nullhomologous knot (see [11]). Knot Floer homologies have also proven to be very useful in knot theoretic applications, the filtration on these groups carrying a lot of geometric information. In [17], we made the observation that the knot Floer homology is not restricted to homologically trivial knots. For $[K]=0$ the knot theoretic information was especially encoded into a filtration constructed using a Seifert surface of $K$. In the case $[K] \neq 0$ this filtration gets lost. The information given by the filtration, however, do not seem to get lost (at least not fully), but are shifted into the Spin ${ }^{c}$-refinements of the groups. We find it natural to also study the groups for $[K] \neq 0$. The first step in this study is to provide tools making the homology groups accessible to computations. Recall, that computations of Heegaard Floer homologies are usually done using surgery exact triangles and adjunction inequalities of the groups involved and of the maps induced by cobordisms. The groups $\widehat{\mathrm{HFK}}$ may be equipped with an adjunction inequality coming from sutured Floer homology (see [4, Theorem 2]). Furthermore, Juhász's work on cobordisms maps for sutured Floer homologies provide a notion of cobordism maps for the $\widehat{\mathrm{HFK}}$-case, as well (see [5]). In [20], we introduced cobordism maps for various versions of knot Floer homology, especially for $\mathrm{HFK}^{\bullet} \circ$ (i.e. $\widehat{\mathrm{HFK}}, \mathrm{HFK}^{\bullet},-$ and $\mathrm{HFK}^{\bullet}, \infty$, see $\S 2.2$ for a definition). 
In this article, we study symmetry properties of knot Floer homology groups $\mathrm{HFK}^{\bullet}, \circ$, adjunction inequalities for $\mathrm{HFK}^{\bullet}, \circ$ and adjunction inequalities for cobordism maps of these groups. After discussing these concepts, we present some implications of these results which are meant as a demonstration how these techniques may be applied in computations. We have to point out that the computational results we present (for the $\widehat{\mathrm{HFK}}$-homology) can be alternatively derived (and some strengthened) from work of Friedl, Juhász and Rasmussen on sutured Floer homology (see [2, Proposition 7.7]).

1.1. Adjunction inequalities. We prove the following result. For the $\widehat{\mathrm{HFK}}$-case, cf. also [4, Theorem 2].

Theorem 1.1. Let $Y$ be a closed, oriented 3-manifold with knot $K$ and $F \subset$ $Y$ a closed, oriented surface with genus $g(F)>0$ such that $\#(K \cap F) \leq 1$ and $\#(F, K) \leq 0$.

(i) If $K$ and $F$ intersect, the non-vanishing of $\operatorname{HFK}^{\bullet}, \circ(Y, K$; $)$ implies that

$$
-\left\langle c_{1}(\mathfrak{s}),[F]\right\rangle \leq 2 g(F)-2 .
$$

(ii) If $K$ and $F$ are disjoint, the non-vanishing of $\operatorname{HFK}^{\bullet}, \circ(Y, K ; \mathfrak{s})$ implies that

$$
\left|\left\langle c_{1}(\mathfrak{s}),[F]\right\rangle\right| \leq 2 g(F)-2 .
$$

The main difficulty here is that the surface $F$ and the knot $K$ might intersect: To elaborate a little on this problem, we would like to note that the adjunction inequalities are set up by constructing a special Heegaard diagram which is adapted to the surface $F$ (see Proposition 3.3). In such diagrams, we obtain a formula for computing the chern class of a $\mathrm{Spin}^{c}$ structure evaluated on $F$ in terms of information encoded in the Heegaard diagram (see Lemma 3.2). However, to make this construction work in the presence of a knot, we have to simultaneously adapt the Heegaard diagram to both the knot and the surface. It turns out that there are basically two cases, namely the two given in the theorem. Multiple intersections have to be resolved by adding handles to the surface $F$. A detailed explanation of such a procedure is given in the proof of Proposition 1.7. To us it seemed peculiar that in case $(i)$ of the theorem the chern class term appears without absolute values. One might think that it should be possible to prove this result with absolute values. However, what we did seems to be the best that can be done. We are able to provide a counterexample to the estimate in (i) with absolute values. We point the interested reader to Remark 2.

Additionally, we prove the following adjunction inequality for cobordism maps between knot Floer homologies. Here, $F^{\bullet}, \circ$ stands for $F^{\bullet, \bullet}, F^{\bullet,-}$ or $F^{\bullet, \infty}$ (see [20]).

Theorem 1.2. Let $Y$ be a closed, oriented 3-manifold with knot $K^{\prime}$ and let $K$ be a homologically trivial knot disjoint from $K^{\prime}$. Denote by $W$ the knot cobordism induced by surgery along a knot $K^{\prime}$. Suppose we are given a 
closed, oriented surface $F \subset W$ with positive genus, $\#\left(I \times K^{\prime} \cap F\right) \leq 1$ and $\#\left(F, I \times K^{\prime}\right) \leq 0$. For $\mathfrak{s} \in \operatorname{Spin}^{c}(W)$ we have the following result:

(i) If $I \times K^{\prime}$ and $F$ intersect, then $[F]^{2} \geq 0$ together with

$$
-\left\langle c_{1}(\mathfrak{s}),[F]\right\rangle-[F]^{2}>2 g(F)-2
$$

implies that $F_{K, \mathfrak{s}}^{\bullet, \circ}=0$.

(ii) If $I \times K$ and $F$ are disjoint, then $[F]^{2} \geq 0$ together with

$$
\left|\left\langle c_{1}(\mathfrak{s}),[F]\right\rangle\right|+[F]^{2}>2 g(F)-2
$$

implies that $F_{K, \mathfrak{s}}^{\bullet, \circ}=0$.

1.2. Symmetries. In $\S 4$ we study symmetries of the knot Floer homologies. The groups $\mathrm{HFK}^{\bullet}, \circ$ share a conjugation symmetry which is given in Proposition 4.1. The knot Floer homology $\widehat{\mathrm{HFK}}$ has some additional symmetries.

Proposition 1.3. Let $Y$ be a closed, oriented 3-manifold with knot $K$ which is homologically essential. Then there is a canonical isomorphism

$$
\phi^{\mathrm{PS}}: \widehat{\operatorname{HFK}}(Y, K ; \mathfrak{s}) \longrightarrow \widehat{\operatorname{HFK}}(Y,-K ; \mathfrak{s}+P D[K])
$$

we will call point-swap isomorphism.

Using the homology class of $[K]$ we define a map $\mathcal{A}$ on the $\operatorname{Spin}^{c}$-structures of $Y$ given by $\mathcal{A}(\mathfrak{s})=\mathfrak{s}+P D[K]$ for $\mathfrak{s} \in \operatorname{Spin}^{c}(Y)$. Using the conjugation map and the map $\mathcal{A}$ we define a new conjugation map we call knot conjugation

$$
\mathcal{N}: \operatorname{Spin}^{c}(Y) \longrightarrow \operatorname{Spin}^{c}(Y)
$$

given by $\mathcal{N}=\mathcal{J} \circ \mathcal{A}$. By applying point-swap symmetry and conjugation symmetry we obtain the following result.

Corollary 1.4. Let $Y$ be a closed, oriented 3-manifold with knot $K \subset Y$. Then for all $\mathfrak{s} \in \operatorname{Spin}^{c}(Y)$ there is an isomorphism

$$
\mathcal{N}_{(Y, K) ; \mathfrak{s}}: \widehat{\operatorname{HFK}}(Y, K ; \mathfrak{s}) \stackrel{\cong}{\operatorname{HFK}}(Y, K ; \mathcal{N}(\mathfrak{s})) \text {. }
$$

This symmetry turns out to be interesting, especially in light of $(i)$ of Theorem 1.1. The knot conjugation symmetry shows that in case $[K] \neq 0$ we have a shifted (or broken) symmetry in $P D[K]$-direction. The missing absolute values in the adjunction inequalities, thus, can be thought of as a manifestation of this shifted (or broken) symmetry. The knot conjugation symmetry carries over to maps induced by cobordisms.

Proposition 1.5. Let $Y$ be a closed, oriented 3-manifold and $K \subset Y a$ knot. Denote by $W$ a cobordism between $(Y, K)$ and $\left(Y^{\prime}, K^{\prime}\right)$. Then, there is a map

$$
\mathcal{N}: \operatorname{Spin}^{c}(W) \longrightarrow \operatorname{Spin}^{c}(W)
$$

with the property that $\mathcal{N} \circ \mathcal{N}=$ id such that

$$
F_{W, \mathfrak{s}}^{\bullet \bullet, \bullet}=\mathcal{N}_{\left(Y^{\prime}, K^{\prime}\right)} \circ F_{W, \mathcal{N}(\mathfrak{s})}^{\bullet \bullet \bullet} \circ \mathcal{N}_{(Y, K)}
$$


for every $\mathfrak{s} \in \operatorname{Spin}^{c}(W)$. We call this knot conjugation symmetry. Furthermore, the maps $F_{W, \mathfrak{s}}^{\bullet, \circ}$ fulfill a conjugation symmetry which says that $F_{W, \mathfrak{s}}^{\bullet, \circ}$ equals $\mathcal{J}_{Y^{\prime}} \circ F_{W, \mathcal{J}(\mathfrak{s})}^{\bullet, \circ} \circ \mathcal{J}_{Y}$.

The map $\mathcal{N}$ on $\operatorname{Spin}^{c}(W)$ will be not be specified, entirely. In the proof of this proposition, we show that $\mathcal{N}$ is a combination of conjugation and a shift with a constant class. However, the discussion in the proof gives all information needed to compute this shift.

1.3. Calculations I. An immediate implication of knot conjugation symmetry is the following result we prove in $\S 5$.

Theorem 1.6. Let $Y$ be a closed, oriented 3-manifold with a knot $K \subset Y$ whose associated homology class $[K]$ cannot be divided by two. Then the rank of the knot Floer homology of the pair $(Y, K)$ is even.

A combination of knot conjugation symmetry and the adjunction inequalities gives the following result.

Proposition 1.7. Let $K, L \subset \mathbb{S}^{3}$ be arbitrary knots and $\Sigma_{S}$ a Seifert surface of $K$ with minimal genus $s g(K)$. Then the group

$$
\widehat{\operatorname{HFK}}\left(\mathbb{S}_{0}^{3}(K), L ; \mathfrak{s}\right)
$$

(a) vanishes for $\mathrm{Spin}^{c}$-structures $\mathfrak{s}$ outside of

$$
\left[-s g(K)-\left\lfloor\frac{\#\left(L \cap \Sigma_{S}\right)}{2}\right\rfloor+1, s g(K)+\left\lfloor\frac{\#\left(L \cap \Sigma_{S}\right)}{2}\right\rfloor-1-l k(K, L)\right]
$$

if $\#\left(L \cap \Sigma_{S}\right)$ is non-zero, odd and $l k(K, L) \neq \#\left(L \cap \Sigma_{S}\right)$, or if the number $\#\left(L \cap \Sigma_{S}\right)$ is even (or zero) and $l k(K, L) \geq 0$,

(b) vanishes for $\mathrm{Spin}^{c}$-structures $\mathfrak{s}$ outside of

$$
\left[-s g(K)-\left\lfloor\frac{\#\left(L \cap \Sigma_{S}\right)}{2}\right\rfloor+1-l k(K, L), s g(K)+\left\lfloor\frac{\#\left(L \cap \Sigma_{S}\right)}{2}\right\rfloor-1\right]
$$

if $\#\left(L \cap \Sigma_{S}\right)$ is non-zero, odd and $l k(K, L)=\#\left(L, \Sigma_{S}\right)$, or if the number $\#\left(L \cap \Sigma_{S}\right)$ is even (or zero) and $l k(K, L) \leq 0$.

We gave this result for knots $K$ and $L$ in the 3 -sphere. However, there is an immediate analogue for knots $K$ and $L$ in an arbitrary closed, oriented 3manifold. In that case, $K$ has to be nullhomologous, but $L$ can be arbitrary. As a special case of this theorem, in combination with knot conjugation symmetry, we almost recover [1, Theorem 3.2] on longitude Floer homology.

Corollary 1.8 (cf. Theorem 1.1. of [1]). For a knot $K \subset \mathbb{S}^{3}$ denote by $s g(K)$ its Seifert genus. The longitude Floer homology $\widehat{\operatorname{HFL}}(K, \mathfrak{s})$ vanishes for $\mathfrak{s}>\operatorname{sg}(K)$ in $1 / 2+\mathbb{Z}$ and for $\mathfrak{s}<-s g(K)$ in $1 / 2+\mathbb{Z}$. Furthermore, we have that

$$
\widehat{\operatorname{HFL}}(K, \mathfrak{s}) \cong \widehat{\operatorname{HFL}}(K,-\mathfrak{s}) \text {. }
$$

Eftekhary's Theorem 3.2 on longitude Floer homology consists of four statements of whom we recover three. 
1.4. Calculations II. When bringing homologically non-trivial knots into the picture the behavior of the knot Floer homologies change: By a result of Ozsváth and Szabó the Heegaard Floer homology $\widehat{\mathrm{HF}}(Y)$ of every closed, oriented 3-manifold is non-zero. If $K$ is a homologically trivial knot in $Y$, there is a spectral sequence from the knot Floer homology $\widehat{\operatorname{HFK}}(Y, K)$ converging to $\widehat{\mathrm{HF}}(Y)$. In consequence, for $K$ homologically trivial, the associated knot Floer homology is non-zero. In contrast to this, in [17] we discovered (and used implicitly) that $\mathbb{S}^{2} \times \mathbb{S}^{1}$ admits a homologically essential knot whose knot Floer homology is completely zero.

Proposition 1.9 (see Proof of Theorem 7.4 of [17]). Denote by $K^{*}$ a fiber of the $\mathbb{S}^{1}$-bundle $\mathbb{S}^{2} \times \mathbb{S}^{1}$. The associated knot Floer homology of the pair $\left(\mathbb{S}^{2} \times \mathbb{S}^{1}, K^{*}\right)$ vanishes, i.e. the group $\widehat{\mathrm{HFK}}\left(\mathbb{S}^{2} \times \mathbb{S}^{1}, K^{*}\right)$ is zero.

Ozsváth and Szabó show in [16] that for a given contact manifold $(Y, \xi)$ there is a contact geometric invariant $\widehat{c}(\xi) \in \widehat{\mathrm{HF}}(-Y)$. Indeed, this invariant is an obstruction to overtwistedness of $\xi$ and it is particularly powerful as demonstrated by results of Lisca and Stipsicz (see $[7,8,9]$ ). We used Proposition 1.9 in [17] to identify a configuration, such that every contact manifold $(Y, \xi)$ with a contact surgery presentation admitting this configuration has vanishing contact invariant $\widehat{c}(\xi)$ (see [17, Theorem 7.4]). In light of this result, finding pairs $(Y, K)$ for which the knot Floer homology is zero will allow us to identify additional configurations in contact surgery diagrams that force the contact element to vanish. Furthermore, we demonstrate in $\S 7$ that results like Proposition 1.9 can be of significant help in calculations when combined with surgery exact triangles. Having a closer look at the example in Proposition 1.9 we see that this is a very special situation. So, it is natural to seek for additional examples among $\mathbb{S}^{1}$-bundles over compact, orientable surfaces. As an application of the adjunction inequalities, in combination with the symmetries we prove that the search will be unsuccessful.

Theorem 1.10. Let $Y$ be a $\mathbb{S}^{1}$-bundle over a closed, oriented surface $\Sigma$ and let $K^{*}$ be a knot isotopic to a fiber. Then $\widehat{\operatorname{HFK}}\left(Y, K^{*}\right)$ is non-zero if and only if $Y$ is not $\mathbb{S}^{2} \times \mathbb{S}^{1}$.

We can interpret this result in such a way that knot Floer homology of a fiber distinguishes the manifold $\mathbb{S}^{2} \times \mathbb{S}^{1}$ from other $\mathbb{S}^{1}$-bundles.

We prove Theorem 1.10 in two steps: In the first step we reprove Proposition 1.9 and give explicit calculations for genus-0 base and non-zero Euler number. In the second step, we prove the general result, i.e. for non-zero genus and arbitrary Euler number. The proof will mainly rely on applying knot conjugation symmetry and a suitable surgery exact triangle. Furthermore, we provide an explicit calculation in $\S 7$ of the case of genus- 1 base and Euler number 0. This serves as a demonstration in what way results like Proposition 1.9 can help to do explicit calculations. 


\section{Preliminaries}

2.1. Heegaard Floer homologies. A 3-manifold $Y$ can be described by a Heegaard diagram, which is a triple $(\Sigma, \boldsymbol{\alpha}, \boldsymbol{\beta})$, where $\Sigma$ is an oriented genus- $g$ surface and $\boldsymbol{\alpha}=\left\{\alpha_{1}, \ldots, \alpha_{g}\right\}, \boldsymbol{\beta}=\left\{\beta_{1}, \ldots, \beta_{g}\right\}$ are two sets of pairwise disjoint simple closed curves in $\Sigma$ called attaching circles. Each set of curves $\boldsymbol{\alpha}$ and $\boldsymbol{\beta}$ is required to consist of linearly independent curves in $H_{1}(\Sigma, \mathbb{Z})$. In the following we will talk about the curves in the set $\boldsymbol{\alpha}$ (resp. $\boldsymbol{\beta}$ ) as $\boldsymbol{\alpha}$-curves (resp. $\boldsymbol{\beta}$-curves). Without loss of generality we may assume that the $\boldsymbol{\alpha}$-curves and $\boldsymbol{\beta}$-curves intersect transversely. To a Heegaard diagram we may associate the triple $\left(\operatorname{Sym}^{g}(\Sigma), \mathbb{T}_{\boldsymbol{\alpha}}, \mathbb{T}_{\boldsymbol{\beta}}\right)$ consisting of the $g$-fold symmetric power of $\Sigma$,

$$
\operatorname{Sym}^{g}(\Sigma)=\Sigma^{\times g} / S_{g}
$$

and the submanifolds $\mathbb{T}_{\boldsymbol{\alpha}}=\alpha_{1} \times \cdots \times \alpha_{g}$ and $\mathbb{T}_{\boldsymbol{\beta}}=\beta_{1} \times \cdots \times \beta_{g}$. We define $\widehat{\mathrm{CF}}(\Sigma, \boldsymbol{\alpha}, \boldsymbol{\beta})$ as the free $\mathbb{Z}_{2}$-module generated by the set $\mathbb{T}_{\boldsymbol{\alpha}} \cap \mathbb{T}_{\boldsymbol{\beta}}$. In the following we will just write $\widehat{\mathrm{CF}}$. For two intersection points $\boldsymbol{x}, \boldsymbol{y} \in \mathbb{T}_{\boldsymbol{\alpha}} \cap \mathbb{T}_{\boldsymbol{\beta}}$ define $\pi_{2}(\boldsymbol{x}, \boldsymbol{y})$ to be the set of homology classes of Whitney discs $\phi: \mathrm{D} \longrightarrow$ $\operatorname{Sym}^{g}(\Sigma)(\mathrm{D} \subset \mathbb{C})$ that connect $\boldsymbol{x}$ with $\boldsymbol{y}$. The map $\phi$ is called Whitney if $\phi(\mathrm{D} \cap\{R e<0\}) \subset \mathbb{T}_{\boldsymbol{\alpha}}$ and $\phi(\mathrm{D} \cap\{R e>0\}) \subset \mathbb{T}_{\boldsymbol{\beta}}$. We call $\mathrm{D} \cap\{R e<0\}$ the $\boldsymbol{\alpha}$-boundary of $\phi$ and $\mathrm{D} \cap\{R e>0\}$ the $\boldsymbol{\beta}$-boundary of $\phi$. Such a Whitney disc connects $\boldsymbol{x}$ with $\boldsymbol{y}$ if $\phi(i)=\boldsymbol{x}$ and $\phi(-i)=\boldsymbol{y}$. Note that $\pi_{2}(\boldsymbol{x}, \boldsymbol{y})$ can be interpreted as the subgroup of elements in $H_{2}\left(\operatorname{Sym}^{g}(\Sigma), \mathbb{T}_{\boldsymbol{\alpha}} \cup\right.$ $\mathbb{T}_{\boldsymbol{\beta}}$ ) represented by discs with appropriate boundary conditions. We endow $\operatorname{Sym}^{g}(\Sigma)$ with a symplectic structure $\omega$. By choosing a path of almost complex structures $\mathcal{J}_{s}$ on $\operatorname{Sym}^{g}(\Sigma)$ suitably (cf. [12]) all moduli spaces of holomorphic Whitney discs are Gromov-compact manifolds. Denote by $\mathcal{M}_{\phi}$ the set of holomorphic Whitney discs in the equivalence class $\phi$, and $\mu(\phi)$ the formal dimension of $\mathcal{M}_{\phi}$. Denote by $\widehat{\mathcal{M}}_{\phi}=\mathcal{M}_{\phi} / \mathbb{R}$ the quotient under the translation action of $\mathbb{R}$ (cf. [12]). Define $H(x, y, k)$ to be the subset of classes in $\pi_{2}(\boldsymbol{x}, \boldsymbol{y})$ that admit moduli spaces of dimension $k$. Fix a point $z \in \Sigma \backslash(\boldsymbol{\alpha} \cup \boldsymbol{\beta})$ and define the map

$$
n_{z}: \pi_{2}(\boldsymbol{x}, \boldsymbol{y}) \longrightarrow \mathbb{Z}, \phi \longmapsto \#\left(\phi,\{z\} \times \operatorname{Sym}^{g-1}(\Sigma)\right) .
$$

A boundary operator $\widehat{\partial}: \widehat{\mathrm{CF}} \longrightarrow \widehat{\mathrm{CF}}$ is given by defining it on the generators $\boldsymbol{x}$ of $\widehat{\mathrm{CF}}$ by

$$
\widehat{\partial} \boldsymbol{x}=\sum_{\boldsymbol{y} \in \mathbb{T}_{\boldsymbol{\alpha}} \cap \mathbb{T}_{\boldsymbol{\beta}}} \sum_{\phi \in H(\boldsymbol{x}, \boldsymbol{y}, 1)} \# \widehat{\mathcal{M}}_{\phi} \cdot U^{n_{z}(\phi)} \boldsymbol{y} .
$$

These homology groups are topological invariants of the manifold $Y$. We would like to note that not all Heegaard diagrams are suitable for defining Heegaard Floer homology; there is an additional condition that has to be imposed called weak admissibility (see [12, Definition 4.10]). 
2.2. Knot Floer Homology. Given a knot $K \subset Y$, we can specify a certain subclass of Heegaard diagrams.

Definition 2.1. A Heegaard diagram $(\Sigma, \boldsymbol{\alpha}, \boldsymbol{\beta})$ is said to be adapted to the knot $K$ if $K$ is isotopic to a knot lying in $\Sigma$ and $K$ intersects $\beta_{1}$ once transversely and is disjoint from the other $\boldsymbol{\beta}$-circles.

Since $K$ intersects $\beta_{1}$ once and is disjoint from the other $\boldsymbol{\beta}$-curves we know that $K$ intersects the core disc of the 2 -handle represented by $\beta_{1}$ once and is disjoint from the others (after possibly isotoping the knot $K$ ). Every pair $(Y, K)$ admits a Heegaard diagram adapted to $K$. Having fixed such a Heegaard diagram $(\Sigma, \boldsymbol{\alpha}, \boldsymbol{\beta})$ we can encode the knot $K$ in a pair of points. After isotoping $K$ onto $\Sigma$, we fix a small interval $I$ in $K$ containing the intersection point $K \cap \beta_{1}$. This interval should be chosen small enough such that $I$ does not contain any other intersections of $K$ with other attaching curves. The boundary $\partial I$ of $I$ determines two points in $\Sigma$ that lie in the complement of the attaching circles, i.e. $\partial I=z-w$, where the orientation of $I$ is given by the knot orientation. This leads to a doubly-pointed Heegaard diagram $(\Sigma, \boldsymbol{\alpha}, \boldsymbol{\beta}, w, z)$. Conversely, a doubly-pointed Heegaard diagram uniquely determines a topological knot class: Connect $w$ with $z$ in the complement of the attaching circles $\boldsymbol{\alpha}$ and $\boldsymbol{\beta} \backslash \beta_{1}$ with an arc $\delta$ that crosses $\beta_{1}$, once. Connect $z$ with $w$ in the complement of $\boldsymbol{\beta}$ using an arc $\gamma$. The union $\delta \cup \gamma$ represents the knot class $K$. The orientation of $K$ is given by orienting $\delta$ such that $\partial \delta=z-w$.

The knot chain complex $\mathrm{CFK}^{\bullet},-(Y, K)$ is the free $\mathbb{Z}_{2}[U]$-module generated by the intersections $\mathbb{T}_{\boldsymbol{\alpha}} \cap \mathbb{T}_{\boldsymbol{\beta}}$. Analogous as above we define $\widehat{\mathcal{M}}_{(\boldsymbol{\alpha}, \boldsymbol{\beta})}^{(i, j)}(\boldsymbol{x}, \boldsymbol{y})$ as the holomorphic Whitney disks connecting $\boldsymbol{x}$ with $\boldsymbol{y}$ such that $\left(n_{z}(\phi), n_{w}(\phi)\right)$ equals $(i, j)$, after modding out the translation action. The boundary operator $\partial_{\boldsymbol{\alpha} \boldsymbol{\beta}}^{\boldsymbol{\bullet}-}$, for $\boldsymbol{x} \in \mathbb{T}_{\boldsymbol{\alpha}} \cap \mathbb{T}_{\boldsymbol{\beta}}$, is defined by

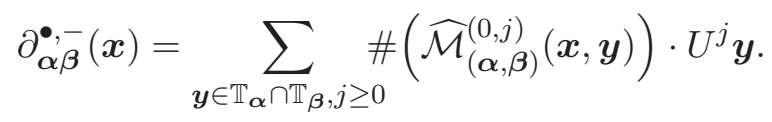

The associated homology theory is denoted by $\operatorname{HFK}^{\bullet},-(Y, K)$. By setting $U=0$ we obtain the theory $\operatorname{HFK}^{\bullet}, \bullet(Y, K)$ which we also denote by $\widehat{\operatorname{HFK}}(Y, K)$. It is also possible to define variants such as $\mathrm{HFK}^{\bullet}++$ and $\operatorname{HFK}^{\bullet}, \infty$. For details we point the reader to [15]. Concerning admissibility, note that for the versions $\mathrm{HFK}^{\bullet}, \circ$ we restrict to doubly pointed Heegaard diagrams $(\Sigma, \boldsymbol{\alpha}, \boldsymbol{\beta}, w, z)$ such that the single pointed diagram $(\Sigma, \boldsymbol{\alpha}, \boldsymbol{\beta}, z)$ is weakly admissible (see [12, Definition 4.10]). Finally, to justify our notation, observe, that it is possible to swap the roles of $z$ and $w$ and so derive knot Floer theories denoted by $\mathrm{HFK}^{-,}, \mathrm{HFK}^{\infty, \bullet}$ and $\mathrm{HFK}^{+, \bullet}$.

For a treatment of cobordism maps between the various versions of knot Floer homology we point the reader to [20, §8 and $\S 9]$. 


\section{A NEW ADJUNCTION INEQUALITY}

Ozsváth and Szabó derived adjunction inequalities for the ordinary Heegaard Floer homology in [13] and gave a knot theoretic version using Seifert surfaces in [11]. An adjunction inequality usually gives an upper bound on the quantity $\left|\left\langle c_{1}(\mathfrak{s}),[F]\right\rangle\right|$ for arbitrary embedded surfaces $F$ and $\operatorname{Spin}^{c}$ structures $\mathfrak{s}$ for which the Floer homology groups are non-zero. Suppose we are given a closed, oriented 3-manifold $Y$ and in it a homologically nontrivial, closed surface $F$ of genus $g(F)$. The main observation to prove our result is to see that we can find a Heegaard diagram which is adapted to the surface.

Lemma 3.1 (Lemma 7.3. of [13]). Suppose $F \subset Y$ is a homologically nontrivial, embedded two-manifold with $g(F)>0$, then $Y$ admits a genus $g$ Heegaard diagram $(\Sigma, \boldsymbol{\alpha}, \boldsymbol{\beta})$, with $g>2 g(F)$, containing a periodic domain $\mathcal{P}$ representing $[F]$, all of whose multiplicities are one or zero. Moreover, $\mathcal{P}$ is a connected surface whose Euler characteristic is equal to $-2 g(F)$, and $\mathcal{P}$ is bounded by $\beta_{1}$ and $\alpha_{2 g+1}$.

In the following, we will call such a Heegaard diagram $F$-adapted. With this Heegaard diagram Ozsváth and Szabó were able to derive the following chern class formula.

Lemma 3.2 (Proposition 7.4. of [13]). If $\boldsymbol{x}=\left\{x_{1}, \ldots, x_{g}\right\}$ is an intersection point, and $z$ is chosen in the complement of the periodic domain $\mathcal{P}$ of Lemma 3.1, then

$$
\left\langle c_{1}\left(\mathfrak{s}_{z}(\boldsymbol{x})\right), \mathcal{H}(\mathcal{P})\right\rangle=2-2 g+2 \#\left\{x_{i} \text { in the interior of } \mathcal{P}\right\} .
$$

With this formula at hand we see that whenever there is an intersection point $\boldsymbol{x}$ whose associated $\operatorname{Spin}^{c}$-structure $\mathfrak{s}_{z}(\boldsymbol{x})$ equals $\mathfrak{s}$ we have that

$$
-\left\langle c_{1}(\mathfrak{s}),[F]\right\rangle \leq 2 g(F)-2
$$

For our purposes we have to see that $F$-adaptedness and adaptedness to a knot $K$ can be achieved simultaneously.

Proposition 3.3. Suppose we are given an embedded surface $F \subset Y$ with $g(F)>0$ which is homologically non-trivial and, further, suppose we are given a knot $K \subset Y$ such that $\#(K \cap F) \leq 1$. Then, there is a F-adapted Heegaard diagram (in the sense of Lemma 3.1) which is adapted to the knot $K$.

Proof. Choose a tubular neighborhood $\nu F=F \times[-1,1]$ of $F$, then the manifold $Y \backslash \nu F$ admits a handle decomposition $\mathcal{D}_{2}$ relative to $\partial(Y \backslash \nu F)$ by 1-handles $\mathrm{h}_{1}^{(3,1)}, \ldots, \mathrm{h}_{l}^{(3,1)}$, 2-handles $\mathrm{h}_{1}^{(3,2)}, \ldots \mathrm{h}_{k}^{(3,2)}$ and one single 3-handle $\mathrm{h}^{(3,3)}$ (cf. [3, p. 104]). The surface $F$ admits a handle decomposition into a single 0 -handle $\mathrm{h}^{(2,0)}$, 1-handles $\mathrm{h}_{1}^{(2,1)}, \ldots, \mathrm{h}_{2 g(F)}^{(2,1)}$ and a single 2-handle $\mathrm{h}^{(2,2)}$. Crossing a 2 -dimensional $k$-handle $\mathrm{h}^{(2, k)}$ with the interval $[-1,1]$ it 
transforms into a 3-dimensional $k$-handle $\underline{\mathrm{h}}^{(3, k)}$. Hence, we obtain a handle decomposition $\mathcal{D}_{1}$ of the tubular neighborhood $\nu F$. Observe, that the $2-$ handle $\underline{\boldsymbol{h}}^{(3,2)}$ comes from $h^{(2,2)}$ which was used to cap-off the boundary of

$$
\mathrm{h}^{(2,0)} \cup_{\partial} \mathrm{h}_{1}^{(2,1)} \cup_{\partial} \ldots \cup_{\partial} \mathrm{h}_{2 g(F)}^{(2,1)} \text {. }
$$

By isotopies of the attaching spheres of the 1-handles we may think the handle $\underline{\underline{h}}^{(3,2)}$ to be attached after the handles $\mathrm{h}_{1}^{(3,1)}, \ldots, \mathrm{h}_{l}^{(3,1)}$. Thus, the pair $\left(\mathcal{D}_{1}, \mathcal{D}_{2}\right)$ induces a Heegaard decomposition, where the handlebody $H_{0}$ is given by the union of $\underline{\mathrm{h}}^{(3,0)}$ with the handles

$$
\underline{\mathrm{h}}^{(3,1)}, \ldots, \underline{\mathrm{h}}_{2 g(F)}^{(3,1)}, \mathrm{h}_{1}^{(3,1)}, \ldots, \mathrm{h}_{l}^{(3,1)} \text {. }
$$

Denote by $\boldsymbol{\beta}_{1}$ the attaching sphere of $\underline{\mathrm{h}}^{(3,2)}$. The curve $\beta_{1}$ bounds a surface $S$, diffeomorphic to $F \backslash D^{2}$, in the Heegaard surface $\Sigma$, since the surface $S$ admits a handle decomposition given in (3.1). The surface $F$ cannot separate and, thus, there has to be a 1-handle, $\mathrm{h}_{2 g+1}^{(3,1)}$-say, that connects $S$ with $\Sigma \backslash D$. Using isotopies of the attaching spheres and handle slides of the 1-handles we again may assume that this is the only 1-handle with this property. The attaching sphere, $\boldsymbol{\alpha}_{2 g+1}$, and $\boldsymbol{\beta}_{1}$ bound a surface whose associated homology element, given by capping it off with the core discs given by $\boldsymbol{\alpha}_{2 g+1}$ and $\boldsymbol{\beta}_{1}$, equals $[F]$. The associated Heegaard diagram is $F$-adapted. To bring in the knot $K$ we have to cover two cases. If $K$ and $F$ are disjoint, we may replace $\mathcal{D}_{1}$ by $\mathcal{D}_{1}^{\prime}$ which is a handle decomposition of $\nu F$ extended to $\nu K$ by an additional 0-handle and 1-handle. Denote by $\mathcal{D}_{2}$ a handle decomposition of $Y \backslash(\nu F \cup \nu K)$ relative to $\partial(\nu F \cup \nu K)$. Observe, that since $\nu F \cup \nu K$ is disconnected there has to be a 1-handle connecting these. Without loss of generality we may think this 1-handle to connect the 0-handles in $\mathcal{D}_{1}^{\prime}$. Thus, the pair $\left(\mathcal{D}_{1}^{\prime}, \mathcal{D}_{2}\right)$ again induces a Heegaard decomposition by the same reasoning as above. The resulting Heegaard diagram is $F$-adapted and $K$-adapted. In case $K$ and $F$ intersect in a unique point we have to be a bit more careful: Denote by $\mathcal{D}_{1}$ a handle decomposition of $\nu F$, with handles denoted like above, such that $\underline{\mathrm{h}}^{(3,0)}$ equals $\nu F \cap \nu K$. With this arrangement we can extend $\mathcal{D}_{1}$ to a decomposition $\mathcal{D}_{1}^{\prime}$ of $\nu K \cup \nu F$ by adding a 1-handle $\mathrm{h}_{*}^{(3,1)}$ given by $\nu K \backslash \underline{\mathrm{h}}^{(3,0)}$. Denote by $\mathcal{D}_{2}$ a handle decomposition of $Y \backslash(\nu K \cup \nu F)$ relative to $\partial(\nu K \cup \nu F)$ with the handles denoted the same way as above. The 2-handle $\underline{\mathrm{h}}^{(3,2)}$ may be thought of as being attached after the 1-handles in the decomposition $\mathcal{D}_{2}$. So, the pair $\left(\mathcal{D}_{1}^{\prime}, \mathcal{D}_{2}\right)$ induces a Heegaard decomposition. We rename the handle $\mathrm{h}_{*}^{(3,1)}$ to $\mathrm{h}_{2 g+1}^{(3,1)}$. The resulting Heegaard diagram is $F$-adapted and $K$-adapted.

Before we prove Theorem 1.1 we have to recall how to recover an oriented knot from a doubly-pointed Heegaard diagram $(\Sigma, \boldsymbol{\alpha}, \boldsymbol{\beta}, w, z)$. Here, it is opportune to look into the Morse-theoretic picture. We obtain a Heegaard diagram from a self-indexing Morse-function $f: Y \longrightarrow \mathbb{R}$. If the associated Heegaard decomposition is $K$-adapted, then $K$ is isotopic to the union of 
two flow lines connecting the index-0 critical point with the index-3 critical point. The Heegaard surface $\Sigma=f^{-1}(3 / 2)$ is oriented such that every flow line intersects $\Sigma$ positively. The two flow lines determining $K$ intersect $\Sigma$ in two points $z$ and $w$. Denote by $\gamma_{z}$ and $\gamma_{w}$ the respective flow lines, then $K$ is isotopic to $\gamma_{z}-\gamma_{w}$. Thus, the knot $K$ when isotoped into $\Sigma$ runs from $z$ to $w$ in the complement of the $\boldsymbol{\beta}$-curves and from $w$ to $z$ in the complement of the $\boldsymbol{\alpha}$-curves.

Proof of Theorem 1.1. Suppose we have chosen an $F$-adapted and $K$-adapted Heegaard diagram $(\Sigma, \boldsymbol{\alpha}, \boldsymbol{\beta})$ with base points $w$ and $z$. Given that the knot Floer homology $\operatorname{HFK}^{\bullet}, \circ(Y, K ; \mathfrak{s})$ is non-zero, there has to be an intersection point $\boldsymbol{x} \in \mathbb{T}_{\boldsymbol{\alpha}} \cap \mathbb{T}_{\boldsymbol{\beta}}$ such that $\mathfrak{s}_{z}(\boldsymbol{x})=\mathfrak{s}$. From the proof of Proposition 3.3 we know that the handle determing the tubular neighborhood of $K$ bounds the periodic domain $\mathcal{P}$ (cf. Lemma 3.1) in case $\#(K \cap F)=1$ and they are disjoint otherwise. Thus, either both $w$ and $z$ lie outside of $\mathcal{P}$ or, depending on the orientation of $K$, one of them lies inside. Observe, that both lie outside of $\mathcal{P}$ in case $F$ and $K$ are disjoint. This means, that there is also a $(-F)$-adapted and $K$-adapted Heegaard diagram with both $w$ and $z$ lying outside of the associated periodic domain. Thus, in this case, we may apply Lemma 3.2 to both get

$$
\begin{aligned}
& 2-2 g(F) \leq\left\langle c_{1}(\mathfrak{s}),[F]\right\rangle \\
& 2-2 g(F) \leq\left\langle c_{1}(\mathfrak{s}),[-F]\right\rangle=-\left\langle c_{1}(\mathfrak{s}),[F]\right\rangle
\end{aligned}
$$

and, consequently,

$$
\left|\left\langle c_{1}(\mathfrak{s}),[F]\right\rangle\right| \leq 2 g(F)-2 .
$$

In case $K$ and $F$ intersect, either $z$ or $w$ lie inside of $\mathcal{P}$. To use the Morse theoretic picture, recall that $K$ is isotopic to the knot $\gamma_{z}-\gamma_{w}$ where $\gamma_{z}$ and $\gamma_{w}$ are the gradient flow lines through $z$ and $w$ of the Morse function defining the Heegaard splitting. Thus, $K$ intersects the Heegaard surface in $z$, positively, and in $w$, negatively. Since $w$ should lie in $\mathcal{P}$ and the orientation of the Heegaard surface and of $F$ coincide, we see that $K$ has to intersect $F$, negatively. With this in place, we may apply Lemma 3.2 to get the desired inequality $(i)$.

Before delving into the proof of Theorem 1.2 we would like to point the reader to [7, Proposition 2.1] and its proof. Lisca and Stipsicz prove an adjunction inequality for cobordism maps $\widehat{F}_{K, \mathfrak{s}}$ in case $b_{1}(Y)=0$. Given that $[F]^{2}=n$ they blow-up the cobordism $W$ at $n$ distinct points in the complement of $F$. Denote by $\widehat{W}$ the new cobordism. Lisca and Stipsicz split up the cobordism into $\widehat{W}_{1}$ and $\widehat{W}_{2}$ where $\widehat{W}=\widehat{W}_{1} \cup_{\partial} \widehat{W}_{2}$. By the composition law of cobordism maps, a homological computation and the adjunction inequalities for the Heegaard Floer groups they show that the map $\widehat{F}_{\widehat{W}}$ factorizes through a trivial Heegaard Floer group. For the homological computation they need the surjectivity of the morphism

$$
\iota_{*}: H_{2}(\widehat{W} ; \mathbb{Z}) \longrightarrow H_{2}(\widehat{W}, Y ; \mathbb{Z})
$$




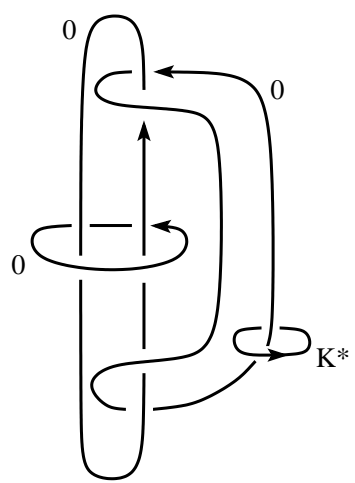

Figure 1. The manifold $T^{3}$ with positively oriented fiber $K^{*}$.

which is a consequence of $b_{1}(Y)=0$. However, by the Mayer-Vietoris sequence

$$
\begin{gathered}
\cdots \longrightarrow H_{1}\left(S^{1}\right) \stackrel{\iota_{1}^{*} \oplus \iota_{2}^{*}}{\longrightarrow} H_{1}(Y \times[0,1]) \oplus H_{1}\left(\mathrm{~h}^{(4,2)}\right) \longrightarrow H_{1}(W) \longrightarrow 0 \\
{\left[S^{1}\right] \longmapsto[K] \oplus 0}
\end{gathered}
$$

we see that surjectivity of $\iota_{*}$ already follows by assuming that $K$ is homologically trivial by comparing with the long exact sequence of the pair $(W, Y)$. Thus, we may follow the lines of their proof but impose the relaxed condition that $[K]=0$ instead of $b_{1}(Y)=0$. Furthermore, in the proof of $[7$, Proposition 2.1] Lisca and Stipsicz use three properties of cobordism maps: The blow-up formula (see [15, Theorem 3.7]), conjugation invariance (see [15, Theorem 3.6]) and the composition law (see [15, Theorem 3.4]). Our proof of the adjunction inequality will consist of showing that for cobordism maps in knot Floer homologies there exists a blow-up formula and a composition law and finally, to see that we can split the knot cobordism $\widehat{W}$ (see $[20$, Definition 8.1]) into two knot cobordisms $\widehat{W}_{1}$ and $\widehat{W}_{2}$.

Proof of Theorem 1.2. The conjugation invariance is given as part of Proposition 1.5 .

Composition law. The composition law essentially requires the associativity of the pair of pants pairing $f_{\boldsymbol{\alpha}, \boldsymbol{\beta} \boldsymbol{\gamma}}^{\bullet, 0}$ defined by counting holomorphic triangles (cf. [20, §8.3]). The necessary statement can be easily derived from the corresponding associativity statement about $\widehat{f}_{\boldsymbol{\alpha}, \boldsymbol{\beta} \boldsymbol{\gamma}}$ by applying $[20$, Theorem 1.2] and [20, Theorem 1.3] with the techniques provided in that paper (see the proof of [20, Theorem 8.7] or [20, Example 4.1]).

Blow-up formula. The proof Ozsváth and Szabó give carries over verbatim to our case with the following assumption imposed: given a knot cobordism $W=([0,1] \times Y, \phi)$ (cf. [20, Definition 8.1]) we blow-up in the complement of the image of $\phi$. We see that for each Spin ${ }^{c}$-structure $\mathfrak{t}$ over 
$Y$ the corresponding map

$$
F_{W ; \mathfrak{s}}^{\bullet, \circ}: \operatorname{HFK}^{\bullet, \circ}(Y, \mathfrak{t}) \longrightarrow \operatorname{HFK}^{\bullet, \circ}(Y, \mathfrak{t})
$$

is the identity for $\left.\mathfrak{s}\right|_{\{0\} \times Y}=\mathfrak{t}$ with $\left\langle c_{1}(\mathfrak{s}), E\right\rangle= \pm 1$ and it vanishes, otherwise.

Splitting up knot cobordisms. We blow up the cobordism $W$ at $n$ distinct points in the complement of the surface $F$ and denote the resulting cobordism by $\widehat{W}$. We take the surface $F$ and pipe them to $n$ exceptional spheres as done in Lisca and Stipsicz's work to obtain a surface $\widehat{F}$. We, further, choose a properly embedded arc $\gamma$ connecting $Y$ with $F$ which is disjoint from $Y$ and $F$ away from its endpoints. A regular neighborhood of $Y \cup \gamma \cup \Sigma$ is denoted by $\widehat{W}_{1}$ and the closure of the complement denoted by $\widehat{W}_{2}$. So far, this is the construction of Lisca and Stipsicz, used in their proof of [7, Proposition 2.1]. In our case, we make a special choice for $\gamma$ : If $[0,1] \times K$ is disjoint from $F$, we choose $\gamma$ to be disjoint from $[0,1] \times K$. If $[0,1] \times K$ and $F$ intersect once, at the point $(t, s)$ say, we set $\gamma=[0, t] \times\{s\}$. Then, for an appropriate regular neighborhood of $Y \cup \gamma \cup F$ the intersection of $[0,1] \times K$ with $\partial \widehat{W}_{1}$ will be a homologically essential embedded knot in $[0,1] \times K$. Hence, the embedded cylinder $[0,1] \times K$ splits into two cylinders, one embedded in $\widehat{W}_{1}$ and one embedded in $\widehat{W}_{2}$. We have that $\partial \widehat{W}_{1}=Y \#\left(\mathbb{S}^{1} \times \widehat{F}\right)$ and we obtain a knot $K^{\prime}$ in this manifold that intersects $\widehat{F}$ once, negatively.

With these remarks done, we follow the lines of the proof of $[7$, Proposition 2.1].

\section{Symmetries}

Symmetries for knot Floer homologies with $[K]=0$ were discussed by Ozsváth and Szabó in [11]. Here, we provide a discussion, which especially covers the case $[K] \neq 0$.

Proof of Proposition 1.3. Given a pair $(Y, K)$ we choose a doubly-pointed Heegaard diagram $\mathcal{H}_{1}=(\Sigma, \boldsymbol{\alpha}, \boldsymbol{\beta}, w, z)$ which is adapted to $K$ and which is extremely-weakly admissible (see [17]). By swapping the points $z$ and $w$ we reverse the orientation of the associated knot, i.e. the Heegaard diagram $\mathcal{H}_{2}=(\Sigma, \boldsymbol{\alpha}, \boldsymbol{\beta}, z, w)$ represents the pair $(Y,-K)$. The chain groups $\widehat{\mathrm{CFK}}\left(\mathcal{H}_{1}\right)$ and $\widehat{\mathrm{CFK}}\left(\mathcal{H}_{2}\right)$ are canonically isomorphic as both are generated by the same set, i.e. both are generated by $\mathbb{T}_{\boldsymbol{\alpha}} \cap \mathbb{T}_{\boldsymbol{\beta}}$. Let

$$
\phi^{\mathrm{PS}}: \widehat{\mathrm{CF}}\left(\mathcal{H}_{1}\right) \longrightarrow \widehat{\mathrm{CF}}\left(\mathcal{H}_{2}\right)
$$

be this canonical isomorphism. Denote by $\widehat{\partial}^{\mathcal{H}_{i}}, i=1,2$ the knot Floer differential associated to the diagrams $\mathcal{H}_{i}$. Both differentials count holomorphic discs $\phi$ with boundary conditions in $\mathbb{T}_{\boldsymbol{\alpha}}$ and $\mathbb{T}_{\boldsymbol{\beta}}$ such that $n_{z}(\phi)=n_{w}(\phi)=0$. Consequently, the equality

$$
\widehat{\partial}^{\mathcal{H}_{1}}=\widehat{\partial}^{\mathcal{H}_{2}}
$$


holds. Hence, $\phi^{\mathrm{PS}}$ induces an isomorphism between the associated knot Floer homologies we will also denote by

$$
\phi^{\mathrm{PS}}: \widehat{\operatorname{HFK}}(Y, K ; \mathfrak{s}) \stackrel{\cong}{\longrightarrow} \widehat{\operatorname{HFK}}\left(Y,-K ; \mathfrak{t}_{\mathfrak{s}}\right)
$$

where $\mathfrak{s}, \mathfrak{t}_{\mathfrak{s}} \in \operatorname{Spin}^{c}(Y)$ are suitable pairs of $\operatorname{Spin}^{c}$-structures. To be precise, given an intersection point $\boldsymbol{x} \in \mathbb{T}_{\boldsymbol{\alpha}} \cap \mathbb{T}_{\boldsymbol{\beta}}$ with $\mathfrak{s}=\mathfrak{s}_{z}(\boldsymbol{x})$, then $\mathfrak{t}_{\mathfrak{s}}$ is the $\operatorname{Spin}^{c}$ structure associated to $\phi^{\mathrm{PS}}(\boldsymbol{x})$. Recall, that $\phi^{\mathrm{PS}}(\boldsymbol{x})=\boldsymbol{x}$, but now $\boldsymbol{x}$ has to be interpreted as a generator of $\widehat{\mathrm{CFK}}\left(\mathcal{H}_{2}\right)$. Since we obtained $\mathcal{H}_{2}$ from $\mathcal{H}_{1}$ by swapping the points $z$ and $w$ we have that

$$
\mathfrak{t}_{\mathfrak{s}}=\mathfrak{s}_{w}(\boldsymbol{x}) .
$$

Applying the formula given in [12, Lemma 2.19] (or looking at the end of its proof) we see that

$$
\mathfrak{s}_{z}(\boldsymbol{x})-\mathfrak{s}_{w}(\boldsymbol{x})=-P D\left[\gamma_{z}-\gamma_{w}\right] .
$$

By definition, $[K]=\left[\gamma_{z}-\gamma_{w}\right]$ and, hence, $\mathfrak{t}_{\mathfrak{s}}=\mathfrak{s}+P D[K]$.

On the $\operatorname{Spin}^{c}$-structures of a manifold $Y$ there is an operation called conjugation given by the following algorithm: Let $[X]$ be a homology class of unit-length vector fields on $Y$ (i.e. a Spin ${ }^{c}$-structure) and let $X$ be a representative. Then we define a map

$$
\mathcal{J}: \operatorname{Spin}^{c}(Y) \longrightarrow \operatorname{Spin}^{c}(Y)
$$

by sending $[X]$ to $[-X]$. The knot Floer homologies fulfill a conjugation symmetry.

Proposition 4.1 (see Proposition 3.9 of [11]). Let $Y$ be a closed, oriented 3 -manifold and $K$ a knot. Then we have that

$$
\operatorname{HFK}^{\bullet, \circ}(Y, K ; \mathfrak{s}) \cong \operatorname{HFK}^{\bullet, \circ}(Y,-K ; \mathcal{J}(\mathfrak{s})) .
$$

We denote by $\mathcal{J}_{Y}$ the associated isomorphism.

Proof. The proof goes exactly as in the case of homologically trivial knots given by Ozsváth and Szabó.

Proof of Corollary 1.3. The isomorphism is given by

$$
\mathcal{N}_{(Y, K) ; \mathfrak{s}}=\mathcal{J}_{Y ; \mathfrak{s}+P D[K]} \circ \phi_{\mathfrak{s}}^{\mathrm{PS}}
$$

or equivalently by $\phi_{\mathfrak{s}}^{\mathrm{PS}} \circ \mathcal{J}_{Y ; \mathfrak{s}}$.

We call the isomorphism $\mathcal{N}_{(Y, K) ; \mathfrak{s}}$ the knot conjugation isomorphism. These morphism allow us to prove that maps induced by cobordisms are symmetric with respect to this knot conjugation.

Proof of Proposition 1.5. We will prove this for cobordisms coming from 2handle attachments. The discussion for 1-handles and 3-handles can done without difficulty. Suppose $W$ is given by attaching a 2-handle along a knot $K^{\prime}$ in the complement of $K$. We define the map $F_{W, \mathfrak{s}}^{\bullet, \bullet}$ (see [20]) in the following way: Let $(\Sigma, \boldsymbol{\alpha}, \boldsymbol{\beta}, w, z)$ be a Heegaard diagram which is $K$-adapted and 
$K^{\prime}$-adapted. The 2-handle attachment along $K^{\prime}$ corresponds to a surgery along $K^{\prime}$. Performing this surgery, we obtain a third set of attaching circles $\boldsymbol{\gamma}$. The doubly-pointed Heegaard triple diagram $(\Sigma, \boldsymbol{\alpha}, \boldsymbol{\beta}, \boldsymbol{\gamma}, w, z)$ determines the cobordism $W$. The map $f_{\boldsymbol{\alpha}, \boldsymbol{\beta} \boldsymbol{\gamma}}^{\boldsymbol{\bullet}, \boldsymbol{r}}$ is defined by counting holomorphic triangles in this diagram and the induced cobordism map (in homology) is denoted by $F_{W}^{\bullet \bullet \bullet}$. Knot conjugation is given as a composition of ordinary conjugation $\mathcal{J}$ and the point-swap isomorphism $\phi^{\mathrm{PS}}$. Both isomorphisms, on the chain level, are the identity. We have to compare the following two maps

$$
\begin{aligned}
& f_{\boldsymbol{\alpha}, \boldsymbol{\beta} \boldsymbol{\gamma}}^{\boldsymbol{\bullet}, \boldsymbol{\beta}}\left(\cdot, \boldsymbol{z}_{\boldsymbol{\beta} \gamma}^{+}\right): \quad \widehat{\mathrm{CFK}}(\Sigma, \boldsymbol{\alpha}, \boldsymbol{\beta}, w, z) \quad \longrightarrow \quad \widehat{\mathrm{CFK}}(\Sigma, \boldsymbol{\alpha}, \boldsymbol{\gamma}, w, z) \\
& f_{\boldsymbol{\beta} \gamma, \boldsymbol{\alpha}}^{\boldsymbol{\bullet}, \boldsymbol{\bullet}}\left(\cdot, \boldsymbol{z}_{\boldsymbol{\gamma} \boldsymbol{\beta}}^{+}\right): \quad \widehat{\mathrm{CFK}}(-\Sigma, \boldsymbol{\beta}, \boldsymbol{\alpha}, z, w) \longrightarrow \widehat{\mathrm{CFK}}(-\Sigma, \boldsymbol{\gamma}, \boldsymbol{\alpha}, z, w)
\end{aligned}
$$

we will first describe in the following: The Heegaard diagram $(\Sigma, \boldsymbol{\beta}, \boldsymbol{\gamma}, w, z)$ is an adapted Heegaard diagram for the pair $\left(\mathbb{S}^{3} \#^{g-1}\left(\mathbb{S}^{2} \times \mathbb{S}^{1}\right), U\right)$ where $U$ is the unknot. There is a subgroup of the associated Heegaard Floer groups we can call the top-dimensional homology (cf. [12] and [13]). Let $\widehat{\Theta}_{\boldsymbol{\beta} \boldsymbol{\gamma}}^{+}$be one of its generators. Recall that there is an intersection point $\boldsymbol{z}_{\boldsymbol{\beta} \boldsymbol{\gamma}}^{+} \in \mathbb{T}_{\boldsymbol{\beta}} \cap \mathbb{T}_{\boldsymbol{\gamma}}$ such that $\left[\boldsymbol{z}_{\boldsymbol{\beta} \boldsymbol{\gamma}}^{+}\right]$is this generator. Suppose we are given $\boldsymbol{x} \in \mathbb{T}_{\boldsymbol{\alpha}} \cap \mathbb{T}_{\boldsymbol{\beta}}$, $\boldsymbol{y} \in \mathbb{T}_{\boldsymbol{\alpha}} \cap \mathbb{T}_{\boldsymbol{\gamma}}$ and a path $\mathcal{J}_{s}$ of admissible almost complex structures. Denote by $\mathcal{M}_{(\boldsymbol{\alpha}, \boldsymbol{\beta}, \boldsymbol{\gamma})}^{\mathcal{J}_{s}}(\boldsymbol{x}, \boldsymbol{z}, \boldsymbol{y})$ the moduli space of $\mathcal{J}_{s}$-holomorphic Whitney triangles connecting $\boldsymbol{x}$ with $\boldsymbol{y}$ through $\boldsymbol{z}$ with boundary conditions in $\mathbb{T}_{\boldsymbol{\alpha}}, \mathbb{T}_{\boldsymbol{\beta}}$ and $\mathbb{T}_{\boldsymbol{\gamma}}$ with Maslox index 0 . The first map is defined via

$$
\left.f_{\boldsymbol{\alpha}, \boldsymbol{\beta} \boldsymbol{\gamma}}^{\boldsymbol{\bullet}, \boldsymbol{e}}\left(\boldsymbol{x}, \boldsymbol{z}_{\boldsymbol{\beta} \gamma}^{+}\right)\right|_{\boldsymbol{y}}=\# \mathcal{M}_{(\boldsymbol{\alpha}, \boldsymbol{\beta}, \boldsymbol{\gamma})}^{\mathcal{J}_{\mathcal{S}}}\left(\boldsymbol{x}, \boldsymbol{z}_{\boldsymbol{\beta} \boldsymbol{\gamma}}^{+}, \boldsymbol{y}\right)
$$

In a similar fashion the second map is defined. Observe, that using conjugation we have an identification

$$
\mathcal{M}_{(\boldsymbol{\alpha}, \boldsymbol{\beta}, \boldsymbol{\gamma})}^{\mathcal{J}_{s}}\left(\boldsymbol{x}, \boldsymbol{z}_{\boldsymbol{\beta} \boldsymbol{\gamma}}^{+}, \boldsymbol{y}\right) \cong \mathcal{M}_{(\boldsymbol{\gamma}, \boldsymbol{\beta}, \boldsymbol{\alpha})}^{-\mathcal{J}_{s}}\left(\boldsymbol{x}, \boldsymbol{z}_{\boldsymbol{\gamma} \boldsymbol{\beta}}^{+}, \boldsymbol{y}\right) .
$$

The intersection point $\boldsymbol{z}_{\beta \gamma}^{+}$can be interpreted as sitting in $(-\Sigma, \boldsymbol{\gamma}, \boldsymbol{\beta})$ and, due to the orientation change of the surface $\Sigma$, the point $\boldsymbol{z}_{\beta \gamma}^{+}$is a representative of $\widehat{\Theta}_{\boldsymbol{\gamma} \boldsymbol{\beta}}^{+}$. Thus, in the new diagram we can interpret the point $\boldsymbol{z}_{\boldsymbol{\beta} \boldsymbol{\gamma}}^{+}$as $\boldsymbol{z}_{\boldsymbol{\gamma} \boldsymbol{\beta}}^{+}$. Hence, we have

$$
\begin{aligned}
\left.f_{\boldsymbol{\alpha}, \boldsymbol{\beta} \boldsymbol{\gamma}}^{\bullet, \bullet}\left(\boldsymbol{x}, \boldsymbol{z}_{\boldsymbol{\beta} \gamma}^{+}\right)\right|_{\boldsymbol{y}} & =\# \mathcal{M}_{(\boldsymbol{\alpha}, \boldsymbol{\beta}, \boldsymbol{\gamma})}^{\mathcal{J}_{s}}\left(\boldsymbol{x}, \boldsymbol{z}_{\boldsymbol{\beta} \gamma}^{+}, \boldsymbol{y}\right)=\# \mathcal{M}_{(\boldsymbol{\gamma}, \boldsymbol{\beta}, \boldsymbol{\alpha})}^{-\mathcal{J}_{s}}\left(\boldsymbol{x}, \boldsymbol{z}_{\boldsymbol{\gamma} \boldsymbol{\beta}}^{+}, \boldsymbol{y}\right) \\
& =\left.f_{\boldsymbol{\beta} \boldsymbol{\gamma}, \boldsymbol{\alpha}}^{\boldsymbol{\bullet}, \boldsymbol{\bullet}}\left(\boldsymbol{x}, \boldsymbol{z}_{\boldsymbol{\gamma} \boldsymbol{\beta}}^{+}\right)\right|_{\boldsymbol{y}} .
\end{aligned}
$$

We have shown that on homology we have

$$
F_{W}^{\bullet, \bullet}=\mathcal{N}_{\left(Y^{\prime}, K^{\prime}\right)} \circ F_{W}^{\bullet, \bullet} \circ \mathcal{N}_{(Y, K)} .
$$

To prove the refined statement given in the proposition, we have to see that there is a map

$$
\mathcal{N}: \operatorname{Spin}^{c}(W) \longrightarrow \operatorname{Spin}^{c}(W)
$$


that refines the equation (4.1). Observe, that knot conjugation is a combination of point-swap symmetry and conjugation symmetry, the former swapping the base points and the latter swapping the roles of $\boldsymbol{\alpha}$ and $\boldsymbol{\beta}$ and altering the surface orientation. From the considerations we have given it is easy to derive that

$$
F_{W, \mathfrak{s}}^{\bullet, \circ}=\mathcal{J}_{Y^{\prime}} \circ F_{W, \mathcal{J}(\mathfrak{s})}^{\bullet, \circ} \circ \mathcal{J}_{Y}
$$

(cf. [15]). To define $\mathcal{N}$, we first have the see in what way swapping base points acts on $\operatorname{Spin}^{c}$-structures. Define $\mathcal{S}_{z} \subset \operatorname{Spin}^{c}(W)$ to be the subset of Spin $^{c}$-structures realized by homotopy classes of Whitney discs in the Heegaard triple diagram $(\Sigma, \boldsymbol{\alpha}, \boldsymbol{\beta}, \boldsymbol{\gamma}, w, z)$ and, correspondingly, define $\mathcal{S}_{w}$ as the subset of $\operatorname{Spin}^{c}$-structures realized by homotopy classes of Whitney triangles in the Heegaard triple diagram $(\Sigma, \boldsymbol{\alpha}, \boldsymbol{\beta}, \boldsymbol{\gamma}, z, w)$. Observe, that our considerations already show that swapping base points induces a map

$$
\eta: \mathcal{S}_{z} \longrightarrow \mathcal{S}_{w}
$$

such that

$$
F_{W, \mathfrak{s}}^{\bullet, \bullet}=\phi_{Y^{\prime}}^{\mathrm{PS}} \circ F_{W, \eta(\mathfrak{s})}^{\bullet, \bullet} \circ \phi_{Y}^{\mathrm{PS}} .
$$

However, we would like to see that $\eta$ extends to a map on the $\operatorname{set}^{\operatorname{Spin}^{c}(W)}$ by showing that $\mathfrak{s}-\eta(\mathfrak{s})$ does not depend on $\mathfrak{s}$. In fact, it is a shift with a constant class. Given a Withney triangle $\phi$, Ozsváth and Szabó in [12, §8.1.4] construct an associated $\operatorname{Spin}^{c}$-structure $\mathfrak{s}_{z}(\phi)$. Performing their construction to determine $\mathfrak{s}_{z}(\phi)$ and $\mathfrak{s}_{w}(\phi)$, we see that these two $\operatorname{Spin}^{c}$-structures will differ only in a tubular neighborhood of $F_{0}^{z} \cup F_{0}^{w}$. We basically apply Ozsváth and Szabó's notation, however, we added the superscript- $z$ and $w$ to $F_{0}$ to indicate the base point used. The construction of both $F_{0}^{z}$ and $F_{0}^{w}$ does not depend on the Whitney triangle $\phi$. Furthermore, the Spin ${ }^{c}$-structures $\mathfrak{s}_{z}(\phi)$ will be independent of $\phi$ in a small tubular neighborhood of $F_{0}^{z}$ and the same holds for $\mathfrak{s}_{w}(\phi)$ and $F_{0}^{w}$. Since we are just looking for Whitney triangles with both $n_{z}(\phi)=n_{w}(\phi)=0$, we will also have that $\mathfrak{s}_{z}(\phi)$ will be independent of $\phi$ at $F_{0}^{w}$ and, vice versa, $\mathfrak{s}_{w}(\phi)$ will be independent of $\phi$ at $F_{0}^{z}$. Thus, the difference class

$$
\mathfrak{s}_{z}(\phi)-\mathfrak{s}_{w}(\phi)
$$

is a constant multiple of the the Poincaré dual of the class $\left[F_{0}^{z} \cup F_{0}^{w}\right]$ in $H_{2}(W, \partial W)$. Thus, there is a homology class $c$ such that $\mathfrak{s}-\eta(\mathfrak{s})=P D[c]$. So, $\eta$ can be extended to a map on $\operatorname{Spin}^{c}(W)$. Hence, defining

$$
\mathcal{N}: \operatorname{Spin}^{c}(W) \longrightarrow \operatorname{Spin}^{c}(W)
$$

as the composition $\mathcal{J} \circ \eta$ we obtain the adequate refinement of equation (4.1).

Remark 1. With more effort it is possible to determine the shifting on Spin $^{c}$-structures of $W$ induced by point-swap symmetry. However, since we do not need an explicit calculation for our purposes, we omitted this step. 


\section{ImPlications to KNOT FloER homology}

Before we continue with applications of the derived symmetries, we would like to remind the reader of the interpretation of $\mathrm{Spin}^{c}$-structures as homology classes of vector fields (cf. [21]): A Spin ${ }^{c}$-structure $\mathfrak{s}$ on a 3-manifold is an equivalence class of unit-length vector fields where two vector fields are defined to be equivalent if they are homotopic outside of a ball. With this description we can characterize a $\operatorname{Spin}^{c}$-structure by the homology class of a link $L_{\mathfrak{s}}$. Furthermore, the first chern class of the Spin ${ }^{c}$-structure and the link are related as follows:

$$
c_{1}(\mathfrak{s})=P D\left[2 \cdot L_{\mathfrak{s}}\right] .
$$

Thus, given a surface $F$ inside a 3 -manifold, the quantity $\left\langle c_{1}(\mathfrak{s}),[F]\right\rangle$ equals twice the intersection number of $L_{\mathfrak{s}}$ with $F$, i.e.

$$
\left\langle c_{1}(\mathfrak{s}),[F]\right\rangle=2 \cdot \#\left(L_{s}, F\right) .
$$

With this at hand we are able to prove the statement of Theorem 1.6.

Proof of Theorem 1.6. This immediately follows from the fact that the knot conjugation

$$
\mathcal{N}: \operatorname{Spin}^{c}(Y) \longrightarrow \operatorname{Spin}^{c}(Y)
$$

is a bijection without fixed points in case $[K]$ cannot be divided by two. If $\widehat{\operatorname{HFK}}(Y, K ; \mathfrak{s})$ has odd rank, then the group $\widehat{\operatorname{HFK}}(Y, K ; \mathcal{N}(\mathfrak{s}))$ has odd rank, too. Supposing that $\mathfrak{s}=\mathcal{N}(\mathfrak{s})=\mathcal{J}(\mathfrak{s})-P D[K]$ we derive that $P D[K]=2 \mathfrak{s}$ which contradicts the assumptions. So, the refined groups come in pairs which both have the same rank.

Indeed, the result given in Theorem 1.6 underpins the difference between the case $[K]=0$ and $[K] \neq 0$.

Proof of Proposition 1.7. The proof combines the adjunction inequalities given in Theorem 1.1, the point-swap symmetry given in Proposition 1.3 and the knot conjugation symmetry given in Proposition 1.5.

First we look at the different conditions posed in $(a)$ and $(b)$. The two conditions $\#\left(L \cap \Sigma_{S}\right)$ is non-zero, odd and $l k(K, L) \neq \#\left(\Sigma \cap \Sigma_{S}\right)$ are equivalent to saying that $\Sigma_{S}$ and $L$ intersect non-trivial, in an odd number of points and at least one of these intersections is negative. On the other hand the conditions $\#\left(L \cap \Sigma_{S}\right)$ is non-zero, odd and $l k(K, L)=\#\left(\Sigma \cap \Sigma_{S}\right)$ means that $L$ and $\Sigma_{S}$ intersect non-trivially, in an odd number of points and all of these intersections are positive. The second set of conditions in (a) and the second set of conditions in (b) can be covered by discussing $L$ and $\Sigma_{S}$, intersecting in an even number of points. Thus, there are basically three cases to cover we will discuss separately.

First suppose $\#\left(\Sigma_{S} \cap L\right)=2 k+1$ is odd and one of these intersection points is negative. Let $\nu L$ be a tubular neighborhood of $L$. The boundary torus $\partial \nu L$ intersects $\Sigma_{S}$ in $2 k+1$ pairwise disjoint, embedded circles. Denote 
by $x_{1}, \ldots, x_{2 k+1}$ the intersection points of $L$ and $\Sigma_{S}$ where the indices are chosen such that, starting at $x_{1}$, traversing along $L$ in the direction given by its orientation, we will meet $\Sigma_{S}$ in order of the intersection points. Without loss of generality we may assume that $x_{1}$ is a negative intersection. Each of the intersection points $x_{i}$ determines a meridian $\mu_{i}$ in $\partial \nu L$. These meridians in turn determine $2 k+1$ cylinders $C_{1}, \ldots, C_{2 k+1}$ in $\partial \nu L$, where $\mu_{j}$ and $\mu_{j+1}$ should bound $C_{j}$. For each $j=1, \ldots, k$, we remove from $\Sigma_{S}$ the disks around $x_{2 j}$ and $x_{2 j+1}$ in which $\nu L$ and $\Sigma_{S}$ meet and glue in the cylinder $C_{2 j}$. In this way we obtain an oriented, embedded surface $\Sigma$ which meets $L$ negatively in one single intersection point. Observe, that we obtained $\Sigma$ from the Seifert surface by adding $k$ one handles. Thus, the group $\widehat{\operatorname{HFK}}\left(\mathbb{S}_{0}^{3}(K), L ; \mathfrak{s}\right)$ vanishes for $\operatorname{Spin}^{c}$-structures $\mathfrak{s}$ which fulfill the following inequality.

$$
-\left\langle c_{1}(\mathfrak{s}),[\Sigma]\right\rangle>2 g(\Sigma)-2=2 s g(K)+2 k-2 .
$$

Since the orientation of $\Sigma$ and $\Sigma_{S}$ agree, the $\operatorname{Spin}^{c}$-structure $\mathfrak{s}=-\lambda \cdot P D[\mu]$ for $\lambda \in \mathbb{Z}^{+}$will fulfill

$$
-\left\langle c_{1}(\mathfrak{s}),[\Sigma]\right\rangle=2 \lambda
$$

Hence, the groups vanish for $\lambda<-s g(K)-k+1$. On the other hand, we may use the surface $-\Sigma$ to say that the groups $\widehat{\operatorname{HFK}}\left(\mathbb{S}_{0}^{3}(K),-L ; \mathfrak{s}\right)$ vanish if

$$
-\left\langle c_{1}(\mathfrak{s}),-[\Sigma]\right\rangle>2 g(\Sigma)-2=2 s g(K)+2 k-2 .
$$

In this case, for $\mathfrak{s}=\epsilon \cdot P D[\mu]$ with $\epsilon \in \mathbb{Z}^{+}$we have that

$$
-\left\langle c_{1}(\mathfrak{s}),-[\Sigma]\right\rangle=2 \epsilon .
$$

Thus, we have vanishing groups for $\epsilon>s g(K)+k-1$. According to the point-swap symmetry given in Proposition 1.3 we see that

$$
\widehat{\operatorname{HFK}}\left(\mathbb{S}_{0}^{3}(K), L ; \mathfrak{s}-P D[L]\right) \cong \widehat{\operatorname{HFK}}\left(\mathbb{S}_{0}^{3}(K),-L ; \mathfrak{s}\right) .
$$

Observe, that $P D[L]=l k(K, L) \cdot P D[\mu]$. Thus, we see that $\widehat{\operatorname{HFK}}\left(\mathbb{S}_{0}^{3}(K), L ; \mathfrak{s}\right)$ vanish for $\mathfrak{s}>s g(K)+k-1-l k(K, L)$. Now, we just have to see that

$$
k=\left\lfloor\frac{\#\left(L \cap \Sigma_{S}\right)}{2}\right\rfloor .
$$

Suppose that $\#(\Sigma \cap L)=2 k+1$ is odd and all intersection points are positive. This case can be covered analogously as the first case. We, again, construct a surface $\Sigma$ as done above by 1-handle additions such that $L$ and $\Sigma$ intersect in a single point. In this case, to apply our adjunction inequality, we have to equip $\Sigma$ with the orientation from $-\Sigma_{S}$. We then proceed as above: By the adjunction inequalities we know that $\widehat{\operatorname{HFK}}\left(\mathbb{S}_{0}^{3}(K), L ; \mathfrak{s}\right)$ vanish for $\operatorname{Spin}^{c}$-structures $\mathfrak{s}$ which fulfill the inequality (5.2): For $\mathfrak{s}=\lambda \cdot P D[\mu]$ with $\lambda \in \mathbb{Z}^{+}$(recall that $\Sigma$ carries the orientation from $-\Sigma_{S}$ ) we have that (5.3) is fulfilled. Hence, the groups vanish for $\lambda>s g(K)+k-1$. Now, looking at $\widehat{\operatorname{HFK}}\left(\mathbb{S}_{0}^{3}(K),-L ; \mathfrak{s}\right)$ we see that these groups vanish for $\mathrm{Spin}^{c}$-structures $\mathfrak{s}$ which fulfill (5.4). So, for $\mathfrak{s}=\epsilon \cdot P D[\mu]$ with $\epsilon \in \mathbb{Z}^{-}$we have that (5.5) is fulfilled. This means that, by application of point-swap symmetry, the 
groups $\widehat{\operatorname{HFK}}\left(\mathbb{S}_{0}^{3}(K), L ; \mathfrak{s}\right)$ also vanish for $\mathfrak{s}<-s g(K)+1-k-l k(K, L)$.

The last case, when $L$ and $\Sigma_{S}$ intersect in an even number of points (or not at all) is the easiest case to cover. By the procedure of handle attachments given at the beginning of the proof we may form a surface $\Sigma$ which is disjoint from $L$, with its genus $g(\Sigma)=s g(K)+k$. Since $\Sigma$ and $L$ are disjoint, part $(b)$ of Theorem 1.1 applies, showing that the groups $\widehat{\operatorname{HFK}}\left(\mathbb{S}_{0}^{3}(K), L ; \mathfrak{s}\right)$ vanish for $\operatorname{Spin}^{c}$-structures outside of the set $I_{1}=[-s g(K)-k+1, s g(K)+k-1]$. The same holds for the groups $\widehat{\operatorname{HFK}}\left(\mathbb{S}_{0}^{3}(K),-L ; \mathfrak{s}\right)$. The latter, by point-swap symmetry, implies that the groups $\widehat{\operatorname{HFK}}\left(\mathbb{S}_{0}^{3}(K), L ; \mathfrak{s}\right)$ vanish for $\mathfrak{s}$ outside of $I_{2}=[-s g(K)-k+1-l k(K, L), s g(K)+k-1-l k(K, L)]$. Thus, the groups vanish for $\mathfrak{s}$ outside of the intersection $I_{1} \cap I_{2}$. In case the linking number of $K$ and $L$ is positive (or zero), $I_{1} \cap I_{2}$ equals the interval given in part $(a)$ and, in case the linking number is negative we obtain the interval in $(b)$.

Suppose we are given a closed, oriented 3-manifold $Y$ and a homologically trivial knot $K$ in it. Further, denote by $\mu$ a meridian of $K$ and by $\widehat{F}$ a closed surface in $Y_{0}(K)$ which is obtained by capping off a Seifert surface of $F$ in $Y$ with the core of the surgery handle. Recall, that the homology of the 0-surgered manifold $Y_{0}(K)$ equals

$$
H^{2}(Y) \oplus \mathbb{Z}
$$

The additional $\mathbb{Z}$-factor we obtain from the 0 -surgery is spanned by the meridian $\mu$ and for a Spin ${ }^{c}$-structure $\mathfrak{s}$ its value in this $\mathbb{Z}$ is given by the quantity

$$
\left\langle c_{1}(\mathfrak{s}),[\widehat{F}]\right\rangle / 2 .
$$

With this preparation it is easy to see that the arguments presented in the proof of Proposition 1.7 can be used to prove an analogue, but more general result, for $K \subset Y$ null-homologous and arbitrary $L \subset Y$ disjoint from $K$. As a special case of Proposition 1.7 and knot conjugation symmetry we almost recover Theorem 1.1. of Eftekhary's paper [1]. Theorem 1.1. states that the longitude Floer homology of a knot in the 3-sphere detects the Seifert genus of the knot in terms of its filtration and that these groups fulfill some kind of conjugation symmetry. The theorem consists of four statements of whom we recover three.

Proof of Corollary 1.8. Given a knot $K$ in the 3-sphere, Eftekhary's longitude Floer homology, by its definition, admits an identification

$$
\widehat{\operatorname{HFL}}(K, \mathfrak{s})=\widehat{\operatorname{HFK}}\left(\mathbb{S}_{0}^{3}(K), \mu ; \mathfrak{s}+1 / 2 \cdot P D[\mu]\right) .
$$

The Spin ${ }^{c}$-shift originates from the fact that Eftekhary filters the longitude Floer homology using the map

$$
\mathfrak{s}=\frac{\mathfrak{s}_{z}+\mathfrak{s}_{w}}{2}
$$


instead of $\mathfrak{s}_{z}$. Since $\mathfrak{s}_{z}=\mathfrak{s}_{w}-P D[\mu]$ (see the discussion in the proof of Proposition 1.3), the identification follows. Given a genus minimizing Seifert surface $\Sigma_{S}$ of $K$, this surface can be capped off in $\mathbb{S}_{0}^{3}(K)$ with a disk. We obtain a closed surface $\widehat{F}$ and we have that $\mu$ intersects $\widehat{F}$ in a single point, positively. So, part (b) of Proposition 1.7 applies, telling us that the knot Floer homology vanishes for $\operatorname{Spin}^{c}$-structures $\mathfrak{s}$ outside of

$$
[-s g(K), s g(K)-1] \text {. }
$$

With the identification between the knot Floer and longitude Floer homology given in (5.6), we see that the latter vanishes for $\mathrm{Spin}^{c}$-structures outside of

$$
[-s g(K)+1 / 2, s g(K)-1 / 2] \text {. }
$$

This concludes the first statement of the corollary. To prove the second statement, recall that by knot conjugation symmetry we have an isomorphism

$$
\mathcal{N}: \widehat{\operatorname{HFK}}\left(\mathbb{S}_{0}^{3}(K), \mu ; \mathfrak{s}\right) \stackrel{\cong}{\operatorname{HFK}}\left(\mathbb{S}_{0}^{3}(K), \mu ; \mathfrak{s}-1\right) .
$$

Using the identification given in equation (5.6) the statement follows.

Remark 2. Recall that part $(i)$ of Theorem 1.1 gives an adjunction inequality without absolute values as given in part $(i i)$ of the theorem. It is worth mentioning that absolute values in case of $(i)$ cannot be achieved in general. The non-vanishing result part of Theorem 3.2. of Eftekhary's paper [1] gives a contradiction. However, even without Eftekhary's result we are able to see that $\widehat{\operatorname{HFK}}\left(\mathbb{S}_{0}^{3}(T), \mu\right)$ gives a counterexample. We will see that this group can be identified with $\widehat{\operatorname{HFK}}\left(T^{3}, K^{*}\right)$, where $K^{*}$ is a fiber of the fibration

$$
\mathbb{S}^{1} \longrightarrow T^{3} \longrightarrow T^{2} \text {. }
$$

Assuming that part $(i)$ of our theorem works with absolute values, this would mean that the former group (and hence the latter) is concentrated in the subgroup associated to the Spin ${ }^{c}$-structure with trivial first chern class. However, using the exact sequences given in $\S 7$ to compute the absolute $\mathbb{Q}$-gradings, this yields a contradiction to the fact that $T^{3}$ admits an orientation-reversing diffeomorphism that fixes the orientation of the knot $K^{*}$ (which implies a certain symmetry in these $\mathbb{Q}$-gradings).

\section{Circle Bundles over the Sphere}

We discuss the case of $\mathbb{S}^{2} \times \mathbb{S}^{1}$ in this section as a first step in the proof of Theorem 1.10. To be more precise, starting from $\mathbb{S}^{2} \times \mathbb{S}^{1}$ we will discuss all circle bundles whose base space is $\mathbb{S}^{2}$ by using surgeries to change the Euler-number of the fibration.

Definition 6.1. We define $\mathbb{F}_{g}^{e}$ to be the $\mathbb{S}^{1}$-bundle with Euler number $e$ and whose base space is a closed, oriented surface of genus $g$.

We start reproving Proposition 1.9 (see also [17, Proof of Theorem 7.4]). 


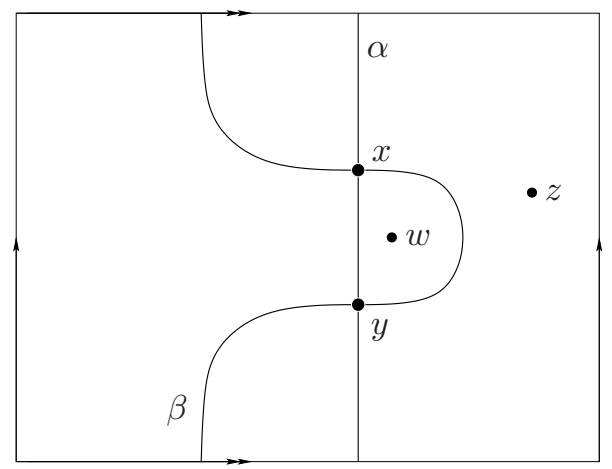

Figure 2. Heegaard diagram adapted to $K^{*}$

Proof of Proposition 1.9. It is not hard to see that Figure 2 pictures a doublypointed Heegaard diagram $\left(T^{2}, \boldsymbol{\alpha}, \boldsymbol{\beta}, w, z\right)$ adapted to the pair $\left(\mathbb{S}^{2} \times \mathbb{S}^{1}, K^{*}\right)$. The chain complex $\widehat{\mathrm{CFK}}(\Sigma, \boldsymbol{\alpha}, \boldsymbol{\beta})$ is generated by the intersection points $\boldsymbol{x}$ and $\boldsymbol{y}$. The boundary operator $\widehat{\partial}^{w}$ is given by:

$$
\begin{aligned}
& \widehat{\partial}^{w} \boldsymbol{x}=\boldsymbol{y} \\
& \widehat{\partial}^{w} \boldsymbol{y}=0 .
\end{aligned}
$$

Thus, the result follows.

For simplicity let us denote by $\mathbb{F}_{g}^{e}$ the $\mathbb{S}^{1}$-bundle over $\Sigma^{g}$ with Euler number $e$. Let $Y$ be a $\mathbb{S}^{1}$-bundle over an oriented surface $\Sigma$. Choose a closed disk $D$ contained in a bundle chart. Then, by choosing sections $\sigma_{1}$ of $\left.Y\right|_{D}$ and $\sigma_{2}$ of $\left.Y\right|_{\Sigma \backslash D}$ we can define the intersection number of $\sigma_{1}$ and $\sigma_{2}$ inside $\left.Y\right|_{\partial D}$. This intersection number is the Euler number of the bundle (see [6]). Using this description it is easy to see that starting with $\mathbb{F}_{g}^{0}$ it is possible to change the Euler number of the bundle using certain surgeries along fibers: Fix a fiber $\gamma$ in $\mathbb{F}_{g}^{0}$. This fiber projects to a point in $\Sigma$. Choose a small disk around that point. Choose a constant section $\sigma_{2}$ over the set $\overline{\Sigma \backslash D}$. This section restricted to $\left.Y\right|_{\partial D} \cong T^{2}$ is a meridian. A surgery along $\gamma$ with coefficient $-1 / e$ provides the necessary modification. We obtain a bundle with Euler number $e$.

Proposition 6.2. Denote by $\mathbb{F}_{0}^{e}$ the $\mathbb{S}^{1}$-bundle over $\mathbb{S}^{2}$ with Euler number $e$ and denote by $K^{*}$ a fiber. Then $H_{1}\left(\mathbb{F}_{0}^{e}\right) \cong \mathbb{Z}_{|e|}$ and for each Spin $^{c}$-structure $\mathfrak{s}$ we have that $\widehat{\operatorname{HFK}}\left(\mathbb{F}_{0}^{e}, K^{*} ; \mathfrak{s}\right) \cong \mathbb{Z}_{2}$.

Proof. Denote by $\gamma$ and $K^{*}$ two distinct fibers of $\mathbb{S}^{2} \times \mathbb{S}^{1}$. To change the Euler number we have to perform a $(-1 / e)$-surgery along $\gamma$. This is pictured in the left of Figure 3. By a slam dunk (cf. [3]) we see that this is the same as the right of Figure 3. Hence, $\mathbb{F}_{0}^{e}$ is the lens space $L(1, e)$. As a matter of fact, the knot $K^{*}$ in $L(1, e)$ generates the first homology group of $L(1, e)$. To 

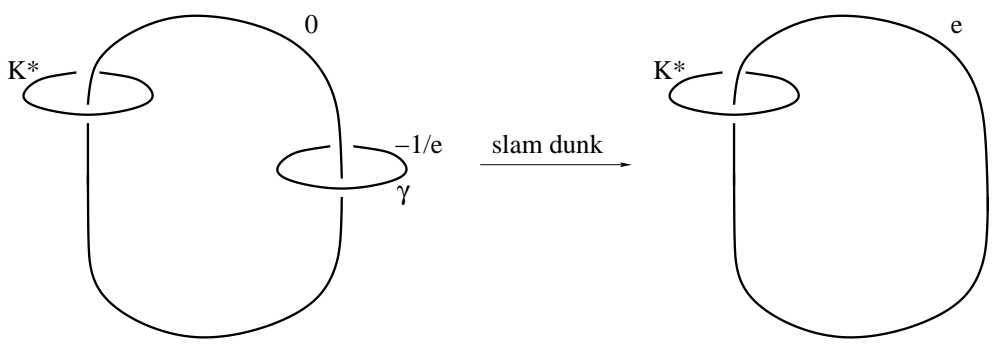

FiguRE 3. Surgery raising the Euler number

compute the knot Floer homology $\widehat{\mathrm{HFK}}\left(\mathbb{F}_{0}^{e}, K^{*}\right)$ we have to find an adapted Heegaard diagram: The 3 -sphere admits a Heegaard decomposition into two full-tori denoted by $H_{1}$ and $H_{2}$. It is possible to isotope the link pictured in the right of Figure 3 such that the components of the link are the cores of the the full-tori $H_{i}, i=1,2$ where $K^{*}$ sits inside $H_{1}$. The gluing of the two handlebodies $H_{i}, i=1,2$ is given by a diffeomorphism $\phi: H_{1} \longrightarrow H_{2}$ which sends $\mu_{1}$, a meridian of $H_{1}$, to $\lambda_{2}$, a longitude of $H_{2}$. Performing the $e$-surgery as indicated in the right of Figure 3, we remove a full-torus $D^{2} \times \mathbb{S}^{1}$ from $H_{2}$ and glue a full-torus $H_{2}^{\prime}$ back in, with a gluing map given by

$$
\begin{aligned}
\psi: \quad \mu^{\prime} & \longmapsto e \cdot \mu_{2}+\lambda_{2} \\
\lambda^{\prime} & \longmapsto-\mu_{2} .
\end{aligned}
$$

We form a new handlebody $H_{1}^{\prime}$ by gluing together $H_{1}$ with $H_{2} \backslash\left(D^{2} \times \mathbb{S}^{1}\right)$. We obtain a Heegaard decomposition $H_{1}^{\prime} \cup_{\partial} H_{2}^{\prime}$ of $\mathbb{F}_{0}^{e}$ with gluing map given by

$$
\mu_{1}^{\prime} \longmapsto \psi^{-1}\left(\lambda_{2}\right)=\mu_{2}^{\prime}+e \cdot \lambda_{2}^{\prime}
$$

This Heegaard decomposition is adapted to the knot $K^{*}$. Figure 4 pictures the associated doubly-pointed Heegaard diagram. As we can see, there are $e$ different intersection points generating the chain complex and all being associated to a different $\mathrm{Spin}^{c}$-structure. Thus, the differential vanishes identically and we have that

$$
\widehat{\operatorname{HFK}}\left(\mathbb{F}_{0}^{e}, K^{*}\right)=\widehat{\operatorname{HF}}(L(1, e))=\mathbb{Z}_{2}^{|e|} .
$$

\section{Three-Dimensional Torus with a Fiber as Knot}

The computation given here serves as a demonstration how results like Proposition 1.9 can be used to simplify computations. In Figure 1 we see a surgery diagram for $T^{3}$. Let us denote by $B_{3}$ the component linked with $K^{*}$, by $B_{2}$ the component linked with $B_{3}$ and by $B_{1}$ the remaining component. The manifold obtained from $\mathbb{S}^{3}$ by performing an $n$-surgery along $B_{1}$, an $m$ surgery along $B_{2}$ and a $k$-surgery along $B_{3}$ should be denoted by $B(n, m, k)$. 


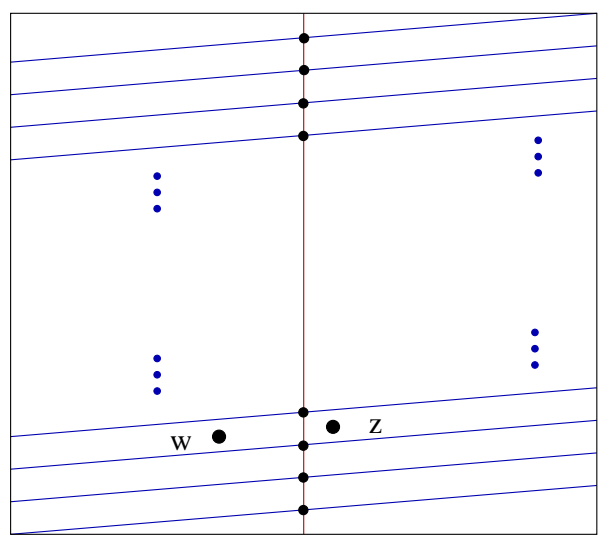

Figure 4. Heegaard diagram adapted to the pair $\left(\mathbb{F}_{0}^{e}, K^{*}\right)$.

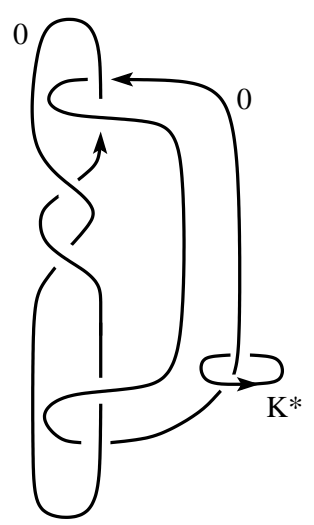

Figure 5. $B(0,1,0)$ is isomorphic to the manifold given here.

The coefficients $n, m, k$ may lie in $\mathbb{Z} \cup\{\infty\}$. The surgery long exact sequence in knot Floer homology gives:

$$
\widehat{\operatorname{HFK}}\left(B(0, \infty, 0), K^{*}\right) \longrightarrow \widehat{\operatorname{HFK}}\left(B(0,0,0), K^{*}\right) \longrightarrow \widehat{\operatorname{HFK}}\left(B(0,1,0), K^{*}\right)
$$

The manifold $B(0, \infty, 0)$ is a connected sum of two $\mathbb{S}^{2} \times \mathbb{S}^{1}$ 's. The knot $K^{*}$ in it corresponds to a fiber of one of the $\mathbb{S}^{2} \times \mathbb{S}^{1}$ components. By $[17$, Corollary 6.8] we know that

$$
\widehat{\operatorname{HFK}}\left(B(0, \infty, 0), K^{*}\right) \cong \widehat{\operatorname{HF}}\left(\mathbb{S}^{2} \times \mathbb{S}^{1}\right) \otimes \widehat{\operatorname{HFK}}\left(\mathbb{S}^{2} \times \mathbb{S}^{1}, K^{*}\right) .
$$

By Proposition 1.9 the group at the right is zero and, hence, the tensor product vanishes. By exactness of the sequence we see that

$$
\widehat{\operatorname{HFK}}\left(\mathbb{F}_{1}^{0}, K^{*}\right)=\widehat{\operatorname{HFK}}\left(B(0,0,0), K^{*}\right) \cong \widehat{\operatorname{HFK}}\left(B(0,1,0), K^{*}\right) .
$$

The manifold $B(0,1,0)$ is isomorphic to the manifold given in Figure 5 . 


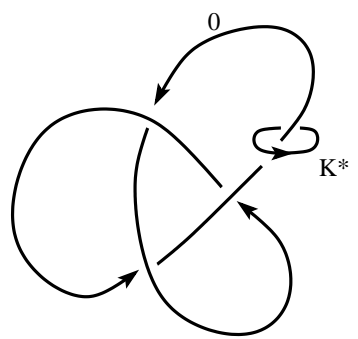

Figure 6. $\widehat{\operatorname{HFK}}\left(T^{3}, K^{*}\right)$ is isomorphic to $\widehat{\operatorname{HFK}}\left(\mathbb{S}_{0}^{3}(T), K^{*}\right)$ with the orientations given in the picture.

We kept track of the knot $K^{*}$. Denote by $W(n, m)$ the manifold obtained from the link given in Figure 5 with surgery coefficient $n$ attached to the knot with the twist and with surgery coefficient $m$ attached to the knot with meridian $K^{*}$. The following sequence is exact:

$$
\widehat{\operatorname{HFK}}\left(W(\infty, 0), K^{*}\right) \longrightarrow \widehat{\operatorname{HFK}}\left(W(0,0), K^{*}\right) \longrightarrow \widehat{\operatorname{HFK}}\left(W(1,0), K^{*}\right)
$$

Since $\left(W(\infty, 0), K^{*}\right)$ equals $\left(\mathbb{S}^{2} \times \mathbb{S}^{1}, K^{*}\right)$ the corresponding knot Floer homology vanishes and, hence,

$$
\widehat{\operatorname{HFK}}\left(W(0,0), K^{*}\right) \cong \widehat{\operatorname{HFK}}\left(W(1,0), K^{*}\right) .
$$

Denote by $T$ the right-handed trefoil knot and denote by $\mathbb{S}_{k}^{3}(T)$ the result of a $k$-surgery along $T$. The pair $\left(W(1,0), K^{*}\right)$ is isomorphic to $\left(\mathbb{S}_{0}^{3}(T), \mu\right)$, where $\mu$ is a meridian of $T$ (see Figure 6). By abuse of notation, we will denote this meridian by $K^{*}$. Consider the following exact sequence:

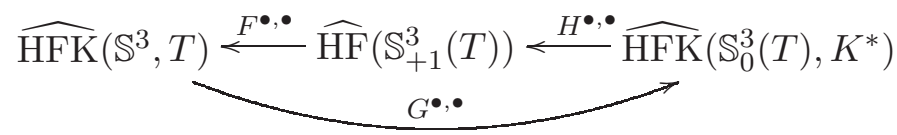

This sequence is derived by either applying the Dehn twist sequence from $[17]$ or applying the surgery exact triangle in knot Floer homology to $\left(\mathbb{S}^{3}, T\right)$, where we start with a surgery along $\gamma$ with framing 0 with $\gamma$ being a push-off of $T$ determining the $(+1)$-framing along $T$. In both cases the map $\widehat{F}$ comes from counting holomorphic triangles in a suitable Heegaard triple diagram. In the Dehn twist case this can be seen by applying the results of [19].

It is worth mentioning, that our results on knot Floer homologies from the previous section already reveals most of the structure of $\widehat{\operatorname{HFK}}\left(\mathbb{S}_{0}^{3}(T), \mu\right)$ : First of all, Theorem 1.6 tells us that the rank of the knot Floer homology is even. It is easy to see that the right-handed trefoil has Seifert genus one. So, according to Proposition 1.7, the group is concentrated in the subgroups associated to the $\operatorname{Spin}^{c}$-structures $\mathfrak{s}_{0}$ and $\mathfrak{s}_{0}-P D[\mu]$, where $\mathfrak{s}_{0}$ is the 
Spin ${ }^{c}$-structure in $\mathbb{S}_{0}^{3}(T)$ with trivial first chern class. The knot conjugation symmetry given in Corollary 1.5 provides an isomorphism between the groups $\widehat{\operatorname{HFK}}\left(\mathbb{S}_{0}^{3}(T), \mu ; \mathfrak{s}_{i}\right), i=1,2$. It remains to compute the rank of the homology.

First of all $\widehat{\mathrm{HF}}\left(\mathbb{S}_{+1}^{3}(T)\right) \cong \mathbb{Z}_{2}$ was calculated in [15, §8.1] (cf. also [10]). The knot Floer homology of the right-handed trefoil was given in [11], it is

$$
\widehat{\operatorname{HFK}}\left(\mathbb{S}^{3}, T\right) \cong\left(\mathbb{Z}_{2}\right)_{(-1 ;-2)} \oplus\left(\mathbb{Z}_{2}\right)_{(0 ;-1)} \oplus\left(\mathbb{Z}_{2}\right)_{(1 ; 0)}
$$

where the subscript $(i ; j)$ means that the corresponding factor sits in the subgroup $\widehat{\mathrm{HFK}}_{j}\left(\mathbb{S}^{3}, T ; i\right)$. Recall, that the maps defined by counting holomorphic triangles, like $F^{\bullet \bullet \bullet}$, can be refined with respect to $\operatorname{Spin}^{c}$-structures of the underlying cobordism, $W$ say, i.e.

$$
F^{\bullet, \bullet}=\sum_{\mathfrak{s} \in \operatorname{Spin}^{c}(W)} F_{\mathfrak{s}}^{\bullet, \bullet}
$$

where

$$
F_{\mathfrak{s}}^{\bullet, \bullet}: \widehat{\operatorname{HF}}\left(\mathbb{S}_{+1}^{3}(T) ;\left.\mathfrak{s}\right|_{\mathbb{S}_{+1}^{3}(T)}\right) \longrightarrow \widehat{\operatorname{HFK}}\left(\mathbb{S}^{3}, T ;\left.\mathfrak{s}\right|_{\mathbb{S}^{3}}\right) .
$$

These maps shift the absolute $\mathbb{Q}$-grading. The grading shift for $F_{\mathfrak{s}}^{\bullet, \bullet}$ is given by

$$
d(\mathfrak{s})=\frac{1}{4}\left(c_{1}(\mathfrak{s})^{2}-2 \chi(W)-3 \sigma(W)\right)
$$

To compute this shift of grading, we have to determine the topology of $W$ and the signature of the intersection form.

Lemma 7.1. The Euler-characteristic of $W$ equals 1 and the signature equals -1 .

Proof. The cobordism $W$ is given by a (-1)-surgery along a meridian $\gamma$ of a meridian $\mu$ of $T$. This means, that $W$ is given by attaching a single 2-handle to $\{1\} \times \mathbb{S}_{+1}^{3}(T)$ of $[0,1] \times \mathbb{S}_{+1}^{3}(T)$. Thus, $H_{0}\left(W, \partial_{-} W\right)=H_{1}\left(W, \partial_{-} W\right)=$ $H_{3}\left(W, \partial_{-} W\right)=H_{4}\left(W, \partial_{-} W\right)=0$ and $H_{2}\left(W, \partial_{-} W\right) \cong \mathbb{Z}$ (cf. [3, p. 111]). We compute the homology groups $H_{k}(W)$ using the long exact sequence of the pair $(W, \partial W)$ where $\partial W=-\mathbb{S}_{+1}^{3}(T) \sqcup \mathbb{S}^{3}$ : We get that $H_{0}(W)=\mathbb{Z}$, $H_{1}(W)=0, H_{2}(W)=\mathbb{Z}, H_{3}(W) \cong \mathbb{Z}$ and $H_{4}(W)=0$. Consequently, $\chi(M)$ equals 1 . We perform a positive Rolfsen twist about $\mu$. This changes the framing of $T$ to +1 , the framing of $\gamma$ to 0 and links $\gamma$ and $T$. In this picture, $\gamma$ is a meridian of $T$. Putting the framing coefficient of $T$ in brackets gives a relative Kirby diagram of $W$. We define a cobordism $X$ obtained from the $D^{4}$ by gluing a 2 -handle along $T$ with framing +1 . The cobordism

$$
Z=X \cup_{\partial} W
$$

is a handlebody and, thus, the intersection form $Q_{Z}$ is given by the linking pairing of the Kirby diagram of $Z$ we obtain by joining the diagrams of $X$ 
and $W$ (cf. [3, Proposition 4.5.11]). The linking matrix is given by

$$
\left(\begin{array}{ll}
1 & 1 \\
1 & 0
\end{array}\right)
$$

after choosing suitable orientations on $T$ and $\gamma$. The homology $H_{2}(Z)$ is generated by surfaces $S_{1}$ and $S_{2}$ we obtain by choosing Seifert surfaces bounding $T$ and $\gamma$ and capping these off with core discs of the handles we attach along $T$ and $\gamma$. Recall, that with respect to this basis the intersection form $Q_{Z}$ is given by (7.4) (cf. [3, Proposition 4.5.11]). The surface $S_{1}$ generates $H_{2}(X)$. The element $\alpha=(-1,1)$ is a primitive element in $H_{2}(Z)$ such that $Q_{Z}\left(\alpha,\left[S_{1}\right]\right)=0$. Hence, the element $\alpha$ generates $H_{2}(W, \partial W) \subset H_{2}(Z)$. It is easy to compute that $Q_{Z}(\alpha, \alpha)=-1$ and, hence, the signature of $W$ is -1 .

Applying the last lemma to the maps $\widehat{F}_{\mathfrak{s}}$ the grading shift equals

$$
d(\mathfrak{s})=\frac{1}{4}\left(c_{1}(\mathfrak{s})^{2}+1\right)
$$

and the values of $c_{1}(\mathfrak{s})^{2}$ are always less or equal to zero. Since, $\widehat{\mathrm{HF}}\left(\mathbb{S}_{+1}^{3}(T)\right)$ is concentrated in degree -2 (cf. $[14, \S 8.1]$ ), we have that

$$
F^{\bullet, \bullet}=\sum_{\mathfrak{s} \in \operatorname{Spin}^{c}(W)} F_{\mathfrak{s}}^{\bullet \bullet \bullet}=\sum_{\mathfrak{s} \in \mathcal{S}} F_{\mathfrak{s}}^{\bullet, \bullet}
$$

where $\mathcal{S}$ are those $\mathfrak{s} \in \operatorname{Spin}^{c}(W)$ such that $c_{1}(\mathfrak{s})^{2}=-1$. If $\mathfrak{t}$ is not contained in $\mathcal{S}$, then

$$
F_{\mathfrak{t}}^{\bullet, \bullet}\left(\widehat{\mathrm{HF}}\left(\mathbb{S}_{+1}^{3}(T)\right)\right) \subset \widehat{\mathrm{HFK}}_{d(\mathfrak{t})-2}\left(\mathbb{S}^{3}, T\right)=0 .
$$

Note that the group is zero, since $d(\mathfrak{t})-2 \notin\{-2,-1,0\}$. Recalling the knot conjugation invariance of the maps induced by cobordisms (see Proposition 1.5), i.e.

$$
F_{\mathfrak{s}}^{\bullet \bullet \bullet}=\mathcal{N}_{\left(\mathbb{S}^{3}, T\right)} \circ F_{\mathcal{N}(\mathfrak{s})}^{\bullet, \bullet} \circ \mathcal{N}_{\left(\mathbb{S}_{+1}^{3}(T), U\right)}
$$

where $U$ denotes the unknot, we see that $F_{\mathfrak{s}}^{\bullet, \bullet}$ vanishes if and only if $F_{\mathcal{N}(\mathfrak{s})}^{\bullet, \bullet}$ vanishes. However, in this case $\mathcal{N}(\mathfrak{s})=\mathcal{J}(\mathfrak{s})$ since both $U$ and $T$ are nullhomologous. Especially observe, that the $\operatorname{Spin}^{c}$-structure of $W$ with trivial chern class is not contained in $\mathcal{S}$. Thus, we can write $\mathcal{S}$ as

$$
\left\{\mathfrak{s}_{-1}, \mathcal{J}\left(\mathfrak{s}_{-1}\right)\right\}
$$

where $c_{1}\left(\mathfrak{s}_{-1}\right)^{2}=c_{1}\left(\mathcal{J}\left(\mathfrak{s}_{-1}\right)\right)^{2}=-1$. Thus,

$$
F^{\bullet, \bullet}=F_{\mathfrak{s}_{-1}}^{\bullet, \bullet}+F_{\mathcal{J}\left(\mathfrak{s}_{-1}\right)}^{\bullet \bullet}
$$

With a Mayer-Vietoris computation we see that $H_{1}\left(\mathbb{S}_{+1}^{3}(T)\right)=0$, since $H_{1}\left(\mathbb{S}^{3} \backslash \nu T\right)$ is generated by a meridian of $T$. Thus,

$$
\begin{aligned}
\widehat{\operatorname{HF}}\left(\mathbb{S}^{3}, T\right) & =\widehat{\operatorname{HF}}\left(\mathbb{S}^{3}, T ; \mathfrak{s}_{0}\right) \\
\widehat{\operatorname{HFK}}\left(\mathbb{S}_{+1}^{3}(T)\right) & =\widehat{\operatorname{HFK}}\left(\mathbb{S}_{+1}^{3}(T) ; \mathfrak{s}_{0}\right)
\end{aligned}
$$


where $\mathfrak{s}_{0}$ are the unique $\operatorname{Spin}^{c}$-structures on both $\mathbb{S}^{3}$ and $\mathbb{S}_{+1}^{3}(T)$ with vanishing first chern class. For these structures we have that $\mathcal{J}\left(\mathfrak{s}_{0}\right)=\mathfrak{s}_{0}$ and, hence, $F_{\mathfrak{s}_{-1}}^{\bullet, \bullet}+F_{\mathcal{J}\left(\mathfrak{s}_{-1}\right)}^{\bullet, \bullet}$ vanishes, i.e. either the summands vanish individually, or their sum vanishes. This finally shows that the map $F^{\bullet}, \bullet$ vanishes identically and from exactness of the sequence (7.3) we get that

$$
\widehat{\operatorname{HFK}}\left(\mathbb{F}_{1}^{0}, K^{*}\right) \cong \widehat{\operatorname{HFK}}\left(\mathbb{S}_{0}^{3}(T), K^{*}\right) \cong \mathbb{Z}_{2}^{4} \text {. }
$$

It is easy to see that $H^{2}\left(T^{3}\right) \cong \mathbb{Z} \oplus \mathbb{Z} \oplus \mathbb{Z}$. Recall, that we denoted by $B_{1}$ the component at the lower-left of Figure $7, B_{2}$ the component at the top of Figure 7 and $B_{3}$ the component at the lower-right. We have chosen $K^{*}$ to be the meridian of $B_{3}$. The knot $B_{1}$ bounds a disk $D$ in $\mathbb{S}^{3}$ which intersects $B_{3}$ in exactly two points. Choose a small tubular neighborhood $\nu B_{3}$ of $B_{3}$, then $D$ cuts $\partial\left(\nu B_{3}\right)$ into two cylinders $Z_{1}$ and $Z_{2}$, i.e. $\partial\left(\nu B_{3}\right) \backslash D=Z_{1} \sqcup Z_{2}$. Pick one of them, $Z_{1}$ say, and define

$$
T=\overline{D \backslash \nu B_{3}} \cup_{\partial} \overline{Z_{1}} .
$$

$T$ is an orientable surface with boundary $B_{1}$. Endow $T$ with an arbitrary orientation. The surface $T$ can be thought of a sitting inside $T^{3}$. Here, the boundary $\partial T$ bounds a disk. We cap off $T$ with a disk to obtain $\widehat{T}$. This torus $\widehat{T}$ is disjoint from $K^{*}$. Observe, that the first homology of $T^{3}$ is generated by meridians $\mu_{i}$ of $B_{i}, i=1, \ldots, 3$. By abuse of notation we will also denote by $\mu_{i}$ the $\mathrm{Spin}^{c}$-structures given by these meridians. By construction the genus of $\widehat{T}$ is one and using the formula (5.1) we see that for any $\operatorname{Spin}^{c}$-structure $\mathfrak{s}=\lambda \mu_{1}$ with $\lambda \neq 0$ we have that

$$
\left|\left\langle c_{1}(\mathfrak{s}),[\widehat{T}]\right\rangle\right|=\left|2 \lambda \cdot \#\left(\mu_{1}, \widehat{T}\right)\right|=2 \lambda>2 g(\widehat{T})-2=0 .
$$

By Theorem 1.1 we see that $\widehat{\operatorname{HFK}}\left(T^{3}, K^{*} ; \mathfrak{s}\right)$ is zero. Using the same construction as above we can construct a torus intersecting $\mu_{2}$ in a single point and which is disjoint from $K^{*}$. The same reasoning as above shows that the knot Floer homology groups are zero, too, for all $\mathfrak{s} \in \mathbb{Z} \backslash\{0\}\left\langle\mu_{2}\right\rangle$. A similar torus can be constructed using the knot $B_{3}$, however, in this case the torus intersects $K^{*}$ in one single point. By Theorem 1.1 we see that for all $\mathfrak{s} \in \mathbb{Z}^{+} \backslash\{0\}\left\langle\mu_{1}\right\rangle$ the associated knot Floer homology vanishes.

Theorem 7.2. For both Spin $^{c}$-structures $\mathfrak{s}_{i}, i=0,1$ the associated knot Floer homology $\widehat{\operatorname{HFK}}\left(T^{3}, K^{*} ; \mathfrak{s}_{i}\right)$ is isomorphic to $\mathbb{Z}_{2} \oplus \mathbb{Z}_{2}$.

Proof. Reversing the orientation of the fiber $K^{*}$, we may apply a similar reasoning as above to see that for all $\mathfrak{s} \in\left(\mathbb{Z} \oplus \mathbb{Z} \oplus \mathbb{Z}^{-}\right) \backslash\{0\}$ the associated group $\widehat{\operatorname{HFK}}\left(T^{3},-K^{*} ; \mathfrak{s}\right)$ is zero. Applying Proposition 1.3 as it was done in the proof of Proposition 1.7, we see that

$$
\widehat{\operatorname{HFK}}\left(T^{3}, K^{*} ; \mathfrak{s}\right) \neq 0
$$

is possible if $\mathfrak{s} \in\left\{\mathfrak{s}_{0}, \mathfrak{s}_{0}-P D[K]\right\}$, only. In the following, denote by $\mathfrak{s}_{1}=$ $\mathfrak{s}_{0}-P D[K]$. With the point-swap symmetry (see Proposition 1.4) we see 


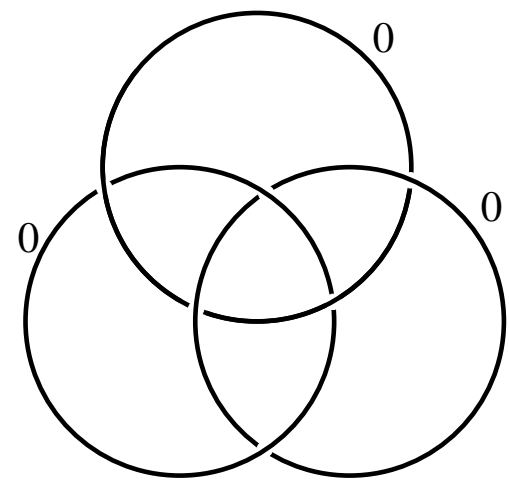

FiguRE 7. Surgery diagram for $T^{3}$.

that both groups $\widehat{\operatorname{HFK}}\left(T^{3}, K^{*} ; \mathfrak{s}_{0}\right)$ and $\widehat{\operatorname{HFK}}\left(T^{3}, K^{*} ; \mathfrak{s}_{1}\right)$ are isomorphic. So, both $\widehat{\operatorname{HFK}}\left(T^{3}, K^{*} ; \mathfrak{s}_{i}\right)$ for $i=0,1$ are isomorphic to $\mathbb{Z}_{2}^{2}$.

\section{Proof of Theorem 1.10}

In Figure 7 we see a surgery diagram for the 3-dimensional torus $T^{3}$. The pictured link is called the Borromean link. To describe the bundle $\Sigma_{2} \times \mathbb{S}^{1}$ we will start with two 3-dimensional tori and perform a fiber-connected sum. It is not hard to see, that a fiber-connected sum of two 3-dimensional tori yields $\Sigma_{2} \times \mathbb{S}^{1}$. Denote by $B \subset \mathbb{S}^{3}$ the Borromean link as pictured in Figure 7. Denote the three components of $B$ by $B_{1}, B_{2}$ and $B_{3}$. Observe, that a meridian of $B_{3}$ say is isotopic to a fiber of $T^{3}$. Now decompose the sphere $\mathbb{S}^{3}$ into two full-tori $H_{0}$ and $H_{1}$, where the gluing between these two tori is given by a map $\phi: \partial H_{0} \longrightarrow \partial H_{1}$ sending a meridian $\mu_{0}$ of $\partial H_{0}$ to a longitude $\lambda_{1}$ of $\partial H_{1}$ and sending a longitude $\lambda_{0}$ of $\partial H_{0}$ to a meridian $\lambda_{1}$ of $\partial H_{1}$. It is possible to isotope the Borromean link $B$ such that it sits in the interior of $H_{0}$ and such that $B_{3}$ is the core of the torus $H_{0}$. In this description $\mu_{0}$, and hence $\lambda_{1}$, are fibers of the torus $T^{3}$. Thus, we may think the fibration of $T^{3}$ at $H_{1}=D^{2} \times \mathbb{S}^{1}$ as being given by the projection

$$
\begin{aligned}
& H_{1}=D^{2} \times \mathbb{S}^{1} \longrightarrow \mathbb{S}^{1} \\
& (r, \theta) \longmapsto \theta \text {. }
\end{aligned}
$$

Now, consider a second 3-dimensional torus. This torus is given by surgery along a Borromean link $B^{\prime} \subset \mathbb{S}^{3}$. We again decompose $\mathbb{S}^{3}$ into two tori $H_{0}^{\prime}$ and $H_{1}^{\prime}$ but now isotope $B^{\prime}$ to sit in the interior of $H_{1}^{\prime}$, the component $B_{3}^{\prime}$ being the core of $H_{1}^{\prime}$. Analogous to our description before, we denote by $\mu_{i}^{\prime}$, $i=0,1$ meridians of $H_{i}^{\prime}$ and by $\lambda_{i}^{\prime}, i=0,1$ longitudes of $H_{i}^{\prime}$. The gluing of the tori $H_{0}^{\prime}$ and $H_{1}^{\prime}$ is given like described above for $H_{0}$ and $H_{1}$. We may think the fibration of the 3-dimensional torus at $H_{0}^{\prime}=D^{2} \times \mathbb{S}^{1}$ as being given by the projection

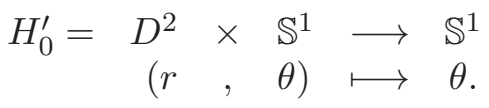




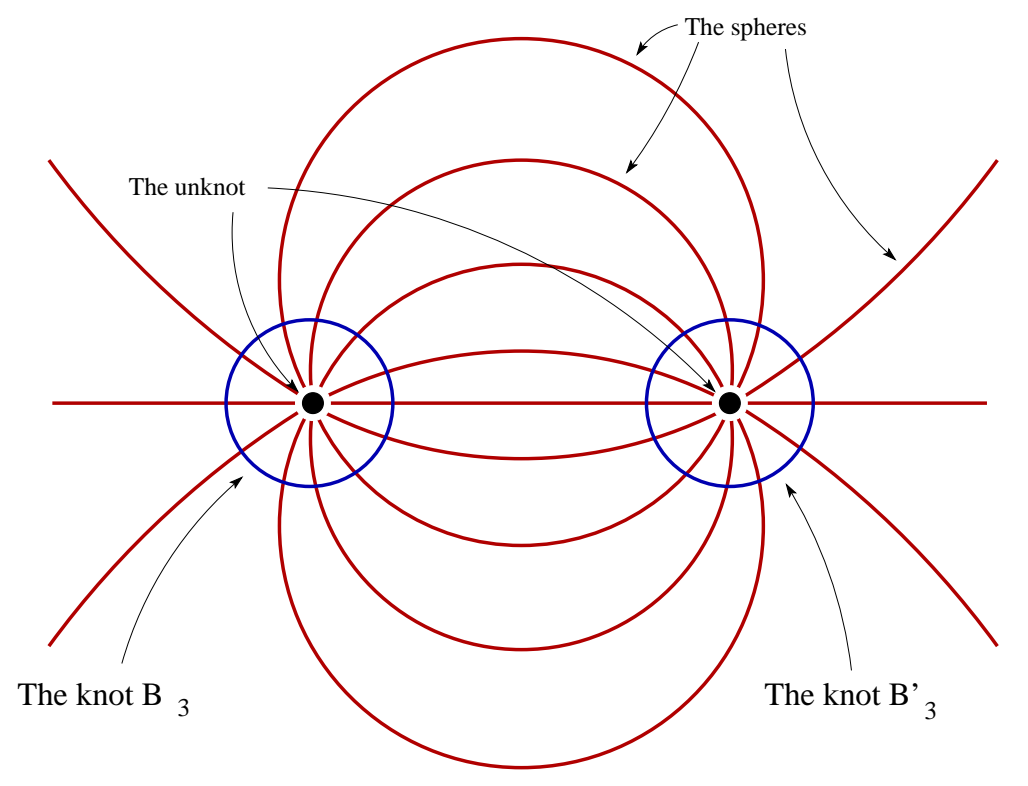

Figure 8. A picture of $\mathbb{S}^{2} \times \mathbb{S}^{1}$ with its spheres $\mathbb{S}^{2} \times\{*\}$ pictured in red.

A fiber-connected sum of the tori can be described by

$$
T^{3} \backslash\left(\operatorname{int}\left(H_{1}\right)\right) \cup_{\partial} T^{3} \backslash\left(\operatorname{int}\left(H_{0}^{\prime}\right)\right)
$$

where the gluing is given by identifying $\lambda_{1}$ with $\lambda_{0}^{\prime}$. Since $\lambda_{1}$ is identified with $\mu_{0}$ and $\lambda_{0}^{\prime}$ identified with $\mu_{0}^{\prime}$ the manifold (8.1) turns into

$$
H_{0} \cup_{\partial} H_{1}^{\prime}
$$

where the gluing is given by sending $\mu_{0}$ to $\mu_{1}^{\prime}$. Thus, $\Sigma_{2} \times \mathbb{S}^{1}$ is obtained from $\mathbb{S}^{2} \times \mathbb{S}^{1}$ by performing 0-surgery along two Borromean knots. The manifold $\mathbb{S}^{2} \times \mathbb{S}^{1}$ is given by performing a 0-surgery along an unknot in $\mathbb{S}^{3}$. Observe, that in $(8.2)$ the knot $B^{3}$ intersects each sphere $\mathbb{S}^{2} \times\{*\}$ in exactly one point, transversely. In the surgery picture of $\mathbb{S}^{2} \times \mathbb{S}^{1}$ we can exactly determine the spheres. In Figure 8 we see the intersection the unit circle in $\mathbb{R}^{2} \times\{0\} \subset \mathbb{R}^{3}$, sitting in the $x y$-plane, with the $x z$-plane. Assume we have performed a 0 -surgery along that knot. In Figure 8 the red lines picture the intersections of the spheres $\mathbb{S}^{2} \times\{*\}$ with the $x z$-plane. The knot $B_{3}$ is a knot which intersects each of the spheres in a single point, transversely. The blue circle around the left point pictures an appropriate knot. Thus, we may think of $B_{3}$ as being that knot. Since $B_{3}^{\prime}$ has intersects each of the $\mathbb{S}^{2} \times\{*\}$ in a single point, too, the blue circle around the right point of Figure 8 can be thought of as begin $B_{3}^{\prime}$. Hence, Figure 9 is a surgery diagram for $\Sigma_{2} \times \mathbb{S}^{1}$. We may inductively continue this process to generate surgery diagrams for each bundle $\Sigma_{g} \times \mathbb{S}^{1}$. Applying a couple of handle slides 


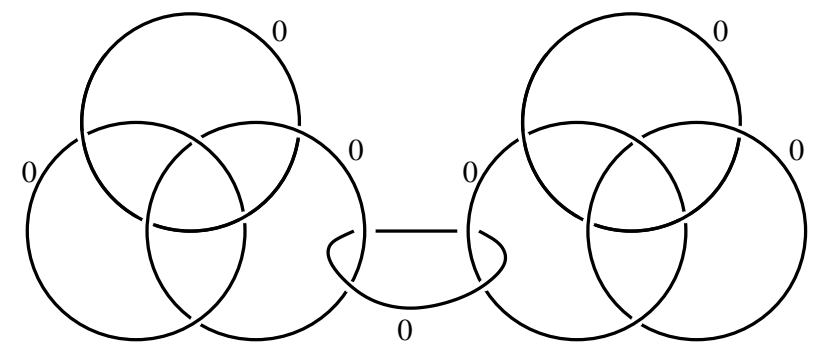

FiguRE 9. A surgery diagram for $\Sigma_{2} \times \mathbb{S}^{1}$.

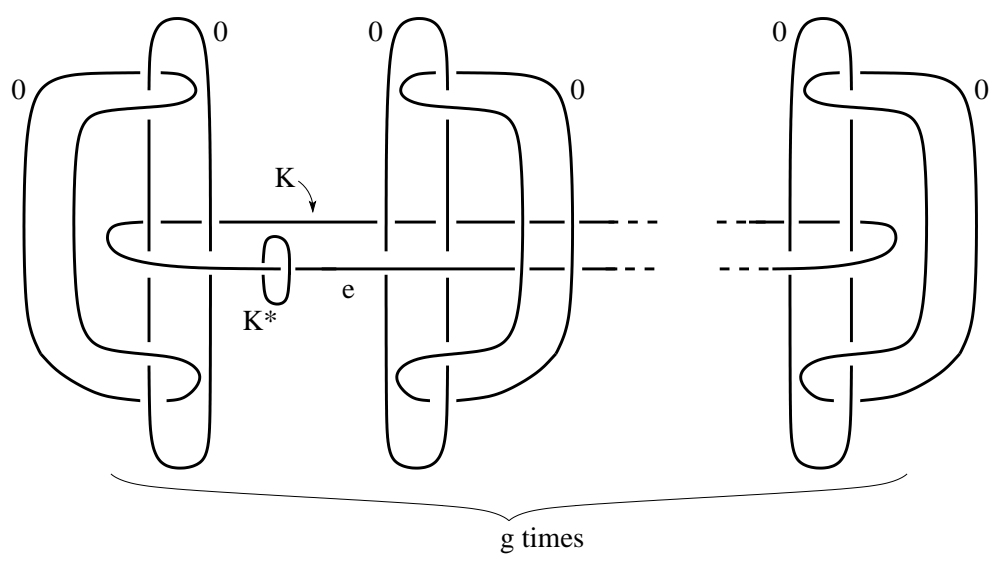

FiguRE 10. A surgery diagram for $\mathbb{F}_{g}^{e}$ with knot $K^{*}$.

and slam dunks we obtain the surgery presentation of $\mathbb{F}_{g}^{e}$ (cf. Definition 6.1) pictured in Figure 10.

Proof of Theorem 1.10. In the following, $K^{*}$ will denote a fiber of $\mathbb{F}_{g}^{e}$ (cf. Definition 6.1). In $\S 6$ we have shown by explicit calculations, that $\widehat{\operatorname{HFK}}\left(\mathbb{F}_{g}^{e}, K^{*}\right)$ is non-zero for $g=0$ and arbitrary non-zero Euler number and checked that it vanishes completely for $g=e=0$. It remains to show that the theorem holds for $g \geq 1$ and $e \in \mathbb{Z}$ : Starting from $Y=\#^{2 g}\left(\mathbb{S}^{2} \times \mathbb{S}^{1}\right)$ we perform an $e$-surgery along $K$ to obtain $\mathbb{F}_{g}^{e}$. We denote by $W$ the induced cobordism. The manifolds $Y$ and $\mathbb{F}_{g}^{e}$ fit into the following surgery exact triangle.

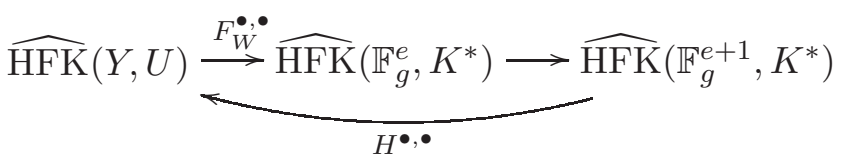

where $U$ denotes the unknot in $Y$. We start with a careful investigation of $\widehat{F}$ in case $e$ is even: It is easy to see that $H_{2}(Y) \cong \mathbb{Z}^{2 g}$ and that $H_{2}\left(\mathbb{F}_{g}^{e}\right) \cong$ $\mathbb{Z}^{2 g} \oplus \mathbb{Z}_{e}$, where we introduced the convention that $\mathbb{Z}_{1}=\mathbb{Z}=\mathbb{Z}_{0}$. Using adjunction inequalities we see that $\widehat{\operatorname{HFK}}(Y, U)$ is concentrated in $\mathfrak{s}_{0}$ and that 
$\widehat{\operatorname{HFK}}\left(\mathbb{F}_{g}^{e}, K^{;} \mathfrak{s}\right)$ can be non-zero for $\mathfrak{s} \in \mathbb{Z}_{e}$, only. First observe, that

$$
\mathfrak{s} \neq \mathcal{N}(\mathfrak{s})
$$

for all $\mathfrak{s} \in \operatorname{Spin}^{c}(W)$ which extend the structures $\mathfrak{s}_{0}$ on $Y$ and $\mathfrak{s}_{*} \in \mathbb{Z}_{e}$ on $\mathbb{F}_{g}^{e}$ : If this were true for a $\operatorname{Spin}^{c}$-structure $\mathfrak{s}$, this would imply that

$$
-\left.\mathfrak{s}\right|_{\mathbb{F}_{g}^{e}}+P D[K]=\left.\mathcal{N}(\mathfrak{s})\right|_{\mathbb{F}_{g}^{e}}=\left.\mathfrak{s}\right|_{\mathbb{F}_{g}^{e}}
$$

which especially means that $[K]$ can be divided by two. This is not possible since $e$ is even. Now observe, that for $\mathfrak{s} \in \operatorname{Spin}^{c}(W)$ extending $\mathfrak{s}_{0}$ on $Y$ we have that

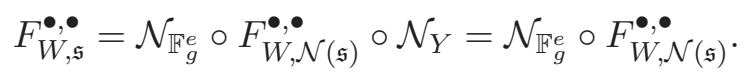

Fix a $\operatorname{Spin}^{c}$-structure $\mathfrak{s}_{*}$ in the $\mathbb{Z}_{e}$-part of $H_{2}\left(\mathbb{F}_{g}^{e}\right)$. Then denote by $\mathcal{S}$ the set of $\operatorname{Spin}^{c}$-structures of $W$ that extend $\mathfrak{s}_{0}$ on $Y$ and $\mathfrak{s}_{*}$ on $\mathbb{F}_{g}^{e}$. The map $F_{W}^{\bullet, \bullet}$ restricted to $\widehat{\operatorname{HFK}}\left(\mathbb{F}_{g}^{e}, K^{*} ; \mathcal{N}\left(\mathfrak{s}_{*}\right)\right)$ can be written as

$$
A=\sum_{\mathfrak{s} \in \mathcal{S}} F_{W, \mathcal{N}(\mathfrak{s})}^{\bullet,}
$$

The map $F_{W}^{\bullet, \bullet}$ restricted to $\widehat{\mathrm{HFK}}\left(\mathbb{F}_{g}^{e}, K^{*} ; \mathfrak{s}_{*}\right)$ can be written as

$$
\sum_{\mathfrak{s} \in \mathcal{S}} F_{W, \mathfrak{s}}^{\bullet, \bullet}=\mathcal{N} \circ A
$$

Furthermore, we know that there is an isomorphism

$$
\widehat{\operatorname{HFK}}\left(\mathbb{F}_{g}^{e}, K^{*} ; \mathfrak{s}\right) \cong \widehat{\operatorname{HFK}}\left(\mathbb{F}_{g}^{e}, K^{*} ; \mathcal{N}(\mathfrak{s})\right) \text {. }
$$

Thus, the map $F_{W}^{\bullet, \bullet}$ restricted to the sum

$$
\widehat{\operatorname{HFK}}\left(\mathbb{F}_{g}^{e}, K^{*} ; \mathfrak{s}\right) \oplus \widehat{\operatorname{HFK}}\left(\mathbb{F}_{g}^{e}, K^{*} ; \mathcal{N}(\mathfrak{s})\right)
$$

equals the map

$$
\mathcal{N} \circ A \oplus A: \widehat{\operatorname{HFK}}\left(Y, U ; \mathfrak{s}_{0}\right) \longrightarrow \widehat{\operatorname{HFK}}\left(\mathbb{F}_{g}^{e}, K^{*} ; \mathfrak{s}\right) \oplus \widehat{\operatorname{HFK}}\left(\mathbb{F}_{g}^{e}, K^{*} ; \mathcal{N}(\mathfrak{s})\right)
$$

with the property that $A(x)=0$ if and only if $\mathcal{N} \circ A(x)=0$. Thus, $\mathcal{N} \circ A \oplus A$ cannot be surjective. Consequently, $F_{W}^{\bullet, \bullet}$ cannot be surjective unless the group $\widehat{\operatorname{HFK}}\left(\mathbb{F}_{g}^{e}, K^{*}\right)$ is zero, completely. In that case, the rank of $\widehat{\operatorname{HFK}}\left(\mathbb{F}_{g}^{e+1}, K^{*}\right)$ is non-zero by exactness of the sequence. Otherwise, since $F_{W}^{\bullet, \bullet}$ is not surjective, it cannot be an isomorphism and, thus, $\widehat{\mathrm{HFK}}\left(\mathbb{F}_{g}^{e+1}, K^{*}\right)$ is non-zero. Our considerations were independent of $g$, we just required that $e$ was even. So, we have shown that the rank of $\widehat{\operatorname{HFK}}\left(\mathbb{F}_{g}^{e}, K^{*}\right)$ for $e$ odd cannot be zero.

Now suppose that $e$ is odd. This means that $e+1$ is even. We want to see, that $H^{\bullet \bullet \bullet}$ cannot be injective: Denote by $W^{\prime}$ the cobordism associated to the map $H^{\bullet} \bullet$. Here, again, the map

$$
\mathcal{N}: \operatorname{Spin}^{c}\left(W^{\prime}\right) \longrightarrow \operatorname{Spin}^{c}\left(W^{\prime}\right)
$$


has no fixed points. Given a $\operatorname{Spin}^{c}$-structure $\mathfrak{s}_{*}$ in the $\mathbb{Z}_{e}$-part of $H_{1}\left(\mathbb{F}_{g}^{e+1}\right)$ and denote by $\mathcal{S}$ the set of $\operatorname{Spin}^{c}$-structures on $W^{\prime}$ that extend $\mathfrak{s}_{*}$ and $\mathfrak{s}_{0}$ on $Y$. We see that

$$
\left.H^{\bullet, \bullet}\right|_{\widehat{\operatorname{HFK}}\left(\mathbb{F}_{g}^{e+1}, K^{*} ; \mathcal{N}\left(\mathfrak{s}_{*}\right)\right)}=\sum_{\mathfrak{s} \in \mathcal{S}} H_{\mathcal{N}(\mathfrak{s})}^{\bullet \bullet \bullet}=: A .
$$

Similarly, since (8.4) is without fixed points, the we have the following equality

$$
\left.H^{\bullet, \bullet}\right|_{\widehat{\operatorname{HFK}}\left(\mathbb{F}_{g}^{e+1}, K^{*} ; \mathfrak{s}_{*}\right)}=\sum_{\mathfrak{s} \in \mathcal{S}} H_{\mathfrak{s}}^{\bullet \bullet \bullet}=A \circ \mathcal{N}
$$

Thus, the map restricted to the groups associated to the Spin ${ }^{c}$-structures $\mathfrak{s}_{*}$ and $\mathcal{N}\left(\mathfrak{s}_{*}\right)$ equals

$$
A \circ \mathcal{N}+A: \widehat{\operatorname{HFK}}\left(\mathbb{F}_{g}^{e+1}, K^{*} ; \mathfrak{s}_{*}\right) \oplus \widehat{\operatorname{HFK}}\left(\mathbb{F}_{g}^{e+1}, K^{*} ; \mathcal{N}\left(\mathfrak{s}_{*}\right)\right) \longrightarrow \widehat{\operatorname{HFK}}\left(Y, U ; \mathfrak{s}_{0}\right) .
$$

This map cannot be injective, since for a non-zero $g \in \widehat{\operatorname{HFK}}\left(\mathbb{F}_{g}^{e+1}, K^{*} ; \mathfrak{s}\right)$ the element $g \oplus-\mathcal{N}(g)$ goes to zero, unless the group $\widehat{\operatorname{HFK}}\left(\mathbb{F}_{g}^{e+1}, K^{*}\right)$ vanishes, completely. If the group vanishes completely, by exactness of the sequence, we know that $\widehat{\operatorname{HFK}}\left(\mathbb{F}_{g}^{e}, K^{*}\right)$ is non-zero. In the non-vanishsing case, however, $H^{\bullet} \bullet$ is not injective and, thus, by exactness of the sequence $\widehat{\operatorname{HFK}}\left(\mathbb{F}_{g}^{e}, K^{*}\right)$ is non-zero. This proves Theorem 1.10.

\section{REFERENCES}

1. E. Eftekhary, Longitude Floer homology and the Whitehead double, Algebr. Geom. Topol. 5 (2005), 1472-2739.

2. S. Friedl and J. Rasmussen and A. Juhasz The decategorification of sutured Floer homology, to appear in J. Topolology.

3. R. E. Gompf and A. I. Stipsicz, 4-Manifolds and Kirby Calculus, Graduate Studies in Mathematics, Vol. 20, American Mathematical Society, 1999.

4. A. Juhász, The sutured Floer homology polytope, Geom. Topol. 14 (2010), 1303-1354.

5. A. Juhász, Cobordisms of sutured manifolds, arXiv:0910.4382v2.

6. K. Niederkrüger, Compact Lie Group Actions on Contact Manifolds, Ph. D. thesis, Universität zu Köln (2005).

7. P. Lisca and A. I. Stipsicz, Ozsváth-Szabó invariants and tight contact manifolds I, Geom. Topol. 8 (2004), 925-945.

8. P. Lisca and A. I. Stipsicz, Ozsváth-Szabó invariants and tight contact manifolds II, J. Diff. Geom. 75 (2007), 109-141.

9. P. Lisca and A. I. Stipsicz, Ozsváth-Szabó invariants and tight contact manifolds III, J. Symplectic Topology 5 (2007), 357-384.

10. B. Ozbagci and A. I. Stipsicz,Surgery on Contact 3-Manifolds and Stein Surfaces, Bolyai Society Mathematical Studies, Vol. 13, Springer-Verlag, (2004).

11. P. Ozsváth and Z. Szabó, Holomorphic disks and knot invariants, Adv. Math. 186 (2004), 58-116.

12. P. Ozsváth and Z. Szabó, Holomorphic disks and topological invariants for closed three-manifolds, Ann. of Math. 159(3) (2004), 1027-1158.

13. P. Ozsváth and Z. Szabó, Holomorphic disks and three-manifold invariants: properties and applications, Ann. of Math. 159(3) (2004), 1159-1245.

14. P. Ozsváth and Z. Szabó, Absolutely graded Floer homologies and intersection forms for four-manifolds with boundary, Adv. in Math. 173 (2003), 179-261. 
15. P. Ozsváth and Z. Szabó, Holomorphic triangles and invariants for smooth fourmanifolds, Adv. in Math. 202 (2006), 326-400.

16. P. Ozsváth and Z. Szabó, Heegaard Floer homologies and contact structures, Duke Math. J. 129(1) (2005), 39-61.

17. B. Sahamie, Dehn twists in Heegaard Floer homology, Algebr. Geom. Topol. 10 (2010), 465-524.

18. B. Sahamie, Introduction to the basics of Heegaard Floer homology, arXiv.org:1003.6041v1 (2010).

19. B. Sahamie, Holomorphic discs and surgery exact triangles, arXiv:1002.4141v1 (2010).

20. B. Sahamie, Whitney polygons, symbol homology and cobordism maps, arXiv:1104.4884v1 (2011).

21. V. Turaev, Torsion invariants of $\operatorname{Spin}^{c}$-structures on 3-manifolds, Math. Research Letters 6 (1997), 679-695.

Mathematisches Institut der LMU MÜnchen, Theresienstrasse 39, 80333 MÜNCHEN GERMANY

E-mail address: sahamie@math.lmu.de

$U R L:$ http://www.math.lmu.de/ sahamie 